Supporting Information for

\title{
Ozonation of Microcystins: Kinetics and Toxicity Decrease
}

\author{
Min Sik Kim, Changha Lee*
}

School of Chemical and Biological Engineering, Institute of Chemical Process (ICP), Seoul National University, 1 Gwanak-ro, Gwanak-gu, Seoul 08826, Republic of Korea

*Corresponding author.

Tel.: +82-2-880-8630, Fax: +82-2-888-7295, E-mail: leechangha@,snu.ac.kr

The supporting information contains 67 pages, 6 texts, 7 tables, and 48 figures. 


\section{Text S1. Reagents}

The chemicals used in this study are as follows: trans-cinnamic acid $\left(\mathrm{CA}, \mathrm{C}_{9} \mathrm{H}_{8} \mathrm{O}_{2}, \geq 99 \%\right.$, Sigma-Aldrich), para-chlorobenzoic acid ( $p \mathrm{CBA}, \mathrm{C}_{7} \mathrm{H}_{5} \mathrm{ClO}_{2}$, 99\%, Sigma-Aldrich), tertbutanol $\left(t-\mathrm{BuOH}, \mathrm{C}_{4} \mathrm{H}_{10} \mathrm{O}, \geq 99.5 \%\right.$, Sigma-Aldrich), benzoic acid (BA, $\geq 99.5 \%$, SigmaAldrich), 2,4-dinitrophenyl hydrazine (DNPH, $\mathrm{C}_{6} \mathrm{H}_{6} \mathrm{~N}_{4} \mathrm{O}_{4}, \geq 97 \%$, Aldrich), potassium oxalate monohydrate $\left(\mathrm{K}_{2} \mathrm{C}_{2} \mathrm{O}_{4} \cdot \mathrm{H}_{2} \mathrm{O}\right.$, 99\%, Sigma-Aldrich), trifluoroacetic acid (TFA, $\mathrm{C}_{2} \mathrm{HF}_{3} \mathrm{O}_{2}$, $>99 \%$, Sigma-Aldrich), $0.1 \%$ formic acid (v/v) in water $\left(\mathrm{CH}_{2} \mathrm{O}_{2}, 0.095-0.105 \%\right.$, Thermo Fisher Scientific), acetonitrile $\left(\mathrm{C}_{2} \mathrm{H}_{3} \mathrm{~N},>99.99 \%\right.$, Honeywell), methanol $\left(\mathrm{CH}_{3} \mathrm{OH},>99.99 \%\right.$, Honeywell), potassium phosphate monobasic $\left(\mathrm{KH}_{2} \mathrm{PO}_{4}, \geq 99 \%\right.$, Simga-Aldrich), potassium phosphate dibasic $\left(\mathrm{K}_{2} \mathrm{HPO}_{4}, \geq 98 \%\right.$, Simga-Aldrich), phosphoric acid $\left(\mathrm{H}_{3} \mathrm{PO}_{4}, 85\right.$ wt.\%, Aldrich), perchloric acid $\left(\mathrm{HClO}_{4}, 70 \%\right.$, Sigma-Aldrich), sodium hydroxide $(\mathrm{NaOH}, \geq 98 \%$, Sigma-Aldrich), sodium tetraborate decahydrate $\left(\mathrm{Na}_{2} \mathrm{~B}_{4} \mathrm{O}_{7} \cdot \mathrm{xH}_{2} \mathrm{O}, \geq 99.5 \%\right.$, Sigma-Aldrich), boric acid $\left(\mathrm{H}_{3} \mathrm{BO}_{3}, \geq 99.5\right.$, Sigma), iron(III) perchlorate hydrate $\left(\mathrm{Fe}\left(\mathrm{ClO}_{4}\right)_{3} \cdot \mathrm{xH}_{2} \mathrm{O}\right.$, crystalline, Sigma), and hydrogen peroxide $\left(\mathrm{H}_{2} \mathrm{O}_{2}, 30\right.$ wt. \% in $\mathrm{H}_{2} \mathrm{O}$, Sigma-Aldrich). DNPH was recrystallized three times using acetonitrile before use. 


\section{Text S2. Determination of second-order rate constants for the reaction of CA with ozone} by stopped-flow spectrometry

Second-order rate constants for the reaction of CA with ozone $\left(k_{\mathrm{O} 3, \mathrm{CA}}\right)$ were determined by measuring the decrease in the concentration of CA in the presence of excess $\mathrm{O}_{3}$; it is much more advantageous in the measurement to monitor the absorbance of $\mathrm{CA}$ than $\mathrm{O}_{3}$, because the molar absorption coefficient of CA is much larger than $\mathrm{O}_{3}$ in $250-300 \mathrm{~nm}$ wavelength $\left(\varepsilon_{280, \mathrm{CA}}\right.$ $=16890 \mathrm{M}^{-1} \mathrm{~cm}^{-1 \mathrm{~S} 1}>\varepsilon_{258, \mathrm{O} 3}=3000 \mathrm{M}^{-1} \mathrm{~cm}^{-1 \mathrm{~S} 2}$ ). The reactions of $\mathrm{CA}$ with $\mathrm{O}_{3}$ are first-order with respect to each reactant, and can be described by Equation S1:

$$
-\mathrm{d}[\mathrm{CA}] / \mathrm{dt}=k_{\mathrm{O} 3, \mathrm{CA}}\left[\mathrm{O}_{3}\right][\mathrm{CA}]
$$

where, $\left[\mathrm{O}_{3}\right]$ and $[\mathrm{CA}]$ represent the concentrations of $\mathrm{O}_{3}$ and $\mathrm{CA}$, respectively. Equation $\mathrm{S} 1$ can be simplified to Equation $\mathrm{S} 2$ in the presence of excess $\mathrm{O}_{3}$ (i.e., $\left[\mathrm{O}_{3}\right]>>[\mathrm{CA}]$ ):

$$
\begin{aligned}
& -\mathrm{d}[\mathrm{CA}] / \mathrm{dt}=k_{\mathrm{obs}}[\mathrm{CA}] \\
& k_{\mathrm{obs}}=k_{\mathrm{O} 3, \mathrm{CA}}\left[\mathrm{O}_{3}\right]
\end{aligned}
$$

where, $k_{\mathrm{obs}}$ is the observed pseudo-first-order rate constant for the reactions of CA with $\mathrm{O}_{3}$ $\left(\mathrm{s}^{-1}\right)$, which can be obtained by measuring the decrease of CA concentration as a function of time.

The time-dependent CA concentration was measured using a stopped-flow spectrometry (SFS) (SFM-4000, Biologic Science Instruments, France) equipped with a UV/vis fiber light source (L10290, Hamamatsu photonics K. K., Japan), a diode array detector (TIDS S 300K, J \& M Analytik AG, Germany), and thermostated water circulator (Polystat 36, Fisher Scientific, France). The SFS system worked with two $10 \mathrm{~mL}$ syringes, each filled with $\mathrm{O}_{3}$ and CA solution (including $t$-BuOH and buffer). For each measurement, $201 \mu \mathrm{L}$ of each reagent in the syringes was injected with a mixing ration of $1: 1$ and a flow rate of $6 \mathrm{~mL} \mathrm{~s}^{-1}$ per syringe. The calculated deadtime was $2.5 \mathrm{~ms}$ based on the total flow rate $\left(12 \mathrm{~mL} \mathrm{~s}^{-1}\right)$ and the dead volume $(30.2 \mu \mathrm{L})$ between rapid mixer and quartz cuvette (1 $\mathrm{cm}$ pathlength). The acquisition of the absorbance data at $280 \mathrm{~nm}$ was performed every millisecond until the CA was completely degraded.

It is important to note that the blank absorbance by $\mathrm{O}_{3}$ should be considered during data processing. To calculate the actual concentration of $\mathrm{CA}$, the absorbance by $\mathrm{O}_{3}$ was 
compensated for by the following data fitting process: i) two fit lines are obtained for the two phases (i.e., decreasing and stationary phases) using OriginPro 8.5 software (OrginLab Co., U.S.A.) (refer to Figure S1a), ii) after subtracting the two fit lines from each other, the actual CA concentrations were calculated using the molar absorption coefficients of CA (refer to Figure S1b). In all experiments, the concentration of CA followed a pseudo-first-order decay. Using Equation $\mathrm{S} 3, k_{\mathrm{O} 3, \mathrm{CA}}$ values were calculated from the slopes of plots of $k_{\mathrm{obs}}$ versus $\left[\mathrm{O}_{3}\right]$.

The effect of $\mathrm{pH}$ on $k_{\mathrm{O} 3, \mathrm{CA}}$ was examined by varying $\mathrm{pH} 2-9$. Considering the $\mathrm{pH}$ dependent species of $\mathrm{CA}\left(\mathrm{p} K_{\mathrm{a}}\right.$ of $\left.\mathrm{CA}=4.4^{\mathrm{S} 3}\right), k_{\mathrm{O}, \mathrm{CA}}$ can be expressed by Equation $\mathrm{S} 4$ :

$$
k_{\mathrm{O} 3, \mathrm{CA}}=k_{\mathrm{O} 3, \mathrm{CA} 1} \alpha_{1}+k_{\mathrm{O} 3, \mathrm{CA} 2} \alpha_{2}
$$

where, $k_{\mathrm{O} 3, \mathrm{CA} i}$ and $\alpha_{i}$ represent the species-specific second-order rate constant and the molar fraction of $\mathrm{CA}_{i}(i=1$ and 2$)$, respectively. The $k_{\mathrm{O}, \mathrm{CA} i}$ values were determined by fitting the experimental data of $k_{\mathrm{O} 3, \mathrm{CA}}$ into Equation $\mathrm{S} 4$, using the least-squares regression function of Microsoft Excel 2016 software (Microsoft Co., U.S.A.). Note that CA concentration at different $\mathrm{pH}$ ranges was calculated using $\mathrm{pH}$-dependent $\varepsilon_{280, \mathrm{CA}}$ values (Figure S2). Both $k_{\mathrm{O} 3, \mathrm{CA}(\mathrm{HA})}$ and $k_{\mathrm{O} 3, \mathrm{CA}(\mathrm{A}-)}$ were determined to be $5.8 \times 10^{4} \mathrm{M}^{-1} \mathrm{~s}^{-1}$ and $7.5 \pm 0.4 \times 10^{5} \mathrm{M}^{-1} \mathrm{~s}^{-1}$, respectively (Table S1, Figures S3a and S4a).

The effect of temperature on $k_{\mathrm{O} 3, \mathrm{CA}}$ was also examined by varying $4-33^{\circ} \mathrm{C}$. The reaction temperature was adjusted to the desired value by thermostat connected to the SFS system. $E_{\mathrm{a}}$ value for the reaction of $\mathrm{CA}$ with $\mathrm{O}_{3}$ were pre-determined to be $19.1 \pm 1.2 \mathrm{~kJ} \mathrm{~mol}^{-1}$ (Table $\mathrm{S} 2$, Figures S3b and S4b). 
Text S3. Determination of the temperature-dependent second-order rate constants for the reaction of $p C B A$ with hydroxyl radical by competition kinetics

Although the original report that investigated rate constants for the reactions of various aromatic compounds with hydroxyl radical $\left({ }^{\bullet} \mathrm{OH}\right)$ provided the second-order rate constant for the reaction of $p \mathrm{CBA}$ with ${ }^{\bullet} \mathrm{OH}\left(k_{\bullet} \mathrm{OH}, p \mathrm{CBA}\right)$ as $5.0 \times 10^{9} \mathrm{M}^{-1} \mathrm{~s}^{-1}$ at $\mathrm{pH} 6-9.4$, no information is

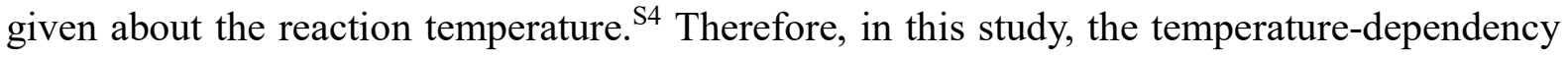
of $k_{\bullet} \mathrm{OH}, p \mathrm{CBA}$ was determined using the competition kinetics (CK) method with BA (as a competitor of $p \mathrm{CBA})$ whose second-rate constant with $\bullet \mathrm{OH}\left(k_{\bullet} \mathrm{OH}, \mathrm{BA}\right)$ and its activation energy $\left(E_{\mathrm{a}}\right)$ are reported to be $6.5 \times 10^{9} \mathrm{M}^{-1} \mathrm{~s}^{-1}$ at $25^{\circ} \mathrm{C}$ and $9.93 \mathrm{~kJ} \mathrm{~mol}^{-1}$, respectively. ${ }^{\mathrm{S} 5}$ Both $p \mathrm{CBA}$ and $\mathrm{BA}$ were not decomposed by UV photolysis during a short illumination time.

The $k_{\bullet} \mathrm{OH}, p \mathrm{CBA}$ values at various temperature conditions can be calculated based on the following CK Equation S5:

$$
\ln \left(\frac{[p C B A]_{0}}{[p C B A]_{t}}\right) / \ln \left(\frac{[B A]_{0}}{[B A]_{t}}\right)=k_{\bullet} \mathrm{OH}, p \mathrm{CBA} / k_{\bullet} \mathrm{OH}, \mathrm{BA}
$$

Where $\mathrm{t}$ is the illumination time. Based on the reported temperature-dependent $k_{\bullet} \mathrm{OH}, \mathrm{BA}$ values, the $k_{\bullet \circ H}, p \mathrm{CBA}$ values were determined from the slope of $\mathrm{CK}$ plot (Figure S6a), and the obtained $k_{\bullet} \mathrm{OH}, p \mathrm{CBA}$ value of $6.0 \pm 0.1 \times 10^{9} \mathrm{M}^{-1} \mathrm{~s}^{-1}$ at $22.3^{\circ} \mathrm{C}$ was comparable to $5.0 \times 10^{9}$ $\mathrm{M}^{-1} \mathrm{~s}^{-1}$ from the literature. ${ }^{\mathrm{S} 4}$ Using the results of Figure S6a, $E_{\mathrm{a}}$ for the reaction of $p \mathrm{CBA}$ with $\bullet \mathrm{OH}$ was determined to be $6.65 \pm 0.47 \mathrm{~kJ} \mathrm{~mol}^{-1}$ from the slope of the Arrhenius plot (Figure S6b). 


\section{Text S4. Correction for UV photolysis of microcystins}

When the $\mathrm{UV} / \mathrm{H}_{2} \mathrm{O}_{2}$ system is applied to generate ${ }^{\bullet} \mathrm{OH}$ for the determination of secondorder rate constant for the reaction of the target microcystin (MC) with ${ }^{\bullet} \mathrm{OH}$, the direct $\mathrm{UV}$ photolysis of the target MC should be corrected. To do this, some experiments using UV without $\mathrm{H}_{2} \mathrm{O}_{2}$ (i.e., UV system) were performed. The portion of direct UV photolysis was corrected by subtracting photolysis rate of the target $\mathrm{MC}$ in the UV system from the degradation rate of the target $\mathrm{MC}$ in the $\mathrm{UV} / \mathrm{H}_{2} \mathrm{O}_{2}$ system, and the detailed procedure is as follows.

The degradation of the target $\mathrm{MC}$ in $\mathrm{UV}$ and $\mathrm{UV} / \mathrm{H}_{2} \mathrm{O}_{2}$ systems can be described by Equations S6 and S7, respectively:

$$
\begin{aligned}
& \left.\frac{\mathrm{d}[\mathrm{MC}]}{\mathrm{dt}}\right|_{\mathrm{UV}}=-I_{0}\left(1-10^{-\varepsilon_{\mathrm{MC}} \mathrm{b}[\mathrm{MC}]}\right) \emptyset_{\mathrm{MC}} \\
& \left.\frac{\mathrm{d}[\mathrm{MC}]}{\mathrm{dt}}\right|_{\mathrm{UV} / \mathrm{H}_{2} \mathrm{O}_{2}}=-I_{0}\left(1-10^{-\varepsilon_{\mathrm{MC}}[\mathrm{MC}]}\right) \emptyset_{\mathrm{MC}}-k \cdot \mathrm{OH}, \mathrm{MC}[\cdot \mathrm{OH}]_{\mathrm{ss}}[\mathrm{MC}]
\end{aligned}
$$

where, $I_{0}$ is the incident photon flow (Einstein $\mathrm{s}^{-1} \mathrm{~L}^{-1}$ ), $\varepsilon_{\mathrm{MC}}$ is the molar absorption coefficient of the target $\mathrm{MC}\left(\mathrm{M}^{-1} \mathrm{~cm}^{-1}\right)$, b is the optical pathlength of the quartz photoreactor $(\mathrm{cm})$, [MC] is the concentration of the target $\mathrm{MC}, \emptyset_{\mathrm{MC}}$ is the quantum yield of photolysis of the target $\mathrm{MC}$, and $\left[{ }^{\bullet} \mathrm{OH}\right]_{\mathrm{ss}}$ is the ${ }^{\bullet} \mathrm{OH}$ concentration at steady state. Equations S6 and S7 can be simplified to Equations S8 and S9 when the absorbance of reaction solution is less than 0.1 (i.e., $\left.\varepsilon_{\mathrm{MC}} \mathrm{b}[\mathrm{MC}]<<0.1\right)$ :

$$
\begin{aligned}
& \left.\frac{\mathrm{d}[\mathrm{MC}]}{\mathrm{dt}}\right|_{\mathrm{UV}} \approx-2.303 I_{0} \varepsilon_{\mathrm{MC}} \mathrm{b} \emptyset_{\mathrm{MC}}[\mathrm{MC}]=-k_{\mathrm{obs}}[\mathrm{MC}],\left(k_{\mathrm{obs}}=2.303 I_{0} \varepsilon_{\mathrm{MC}} \mathrm{b} \emptyset_{\mathrm{MC}}\right) \\
& \left.\frac{\mathrm{d}[\mathrm{MC}]}{\mathrm{dt}}\right|_{\mathrm{UV} / \mathrm{H}_{2} \mathrm{O}_{2}}=-k_{\mathrm{obs}}[\mathrm{MC}]-k_{\mathrm{obs}}^{*}[\mathrm{MC}],\left(k_{\mathrm{obs}}^{*}=k_{\cdot \mathrm{OH}, \mathrm{MC}}[\cdot \mathrm{OH}]_{\mathrm{ss}}\right)
\end{aligned}
$$

where, $k_{\mathrm{obs}}$ and $k_{\mathrm{obs}}^{*}$ are the pseudo-first order rate constant of degradation of the target MC by $\mathrm{UV}$ and ${ }^{\bullet} \mathrm{OH}$, respectively, which can be obtained by measuring the decrease of the target $\mathrm{MC}$ concentration as a function of time in $\mathrm{UV}$ and $\mathrm{UV} / \mathrm{H}_{2} \mathrm{O}_{2}$ systems (Equations $\mathrm{S} 10$ and S11).

$$
\ln \left(\frac{[\mathrm{MC}]_{0}}{[\mathrm{MC}]}\right)_{\mathrm{UV}}=k_{\mathrm{obs}} \mathrm{t}
$$




$$
\ln \left(\frac{[\mathrm{MC}]_{0}}{[\mathrm{MC}]}\right)_{{\mathrm{UV} / \mathrm{H}_{2} \mathrm{O}_{2}}}=\left(k_{\mathrm{obs}}+k_{\mathrm{obs}}^{*}\right) \mathrm{t}
$$

Therefore, the degradation rate of the target $\mathrm{MC}$ by ${ }^{\circ} \mathrm{OH}$ can be given by Equation $\mathrm{S} 12$ by subtracting Equation S10 from Equation S11:

$$
\ln \left(\frac{[\mathrm{MC}]_{0}}{[\mathrm{MC}]}\right)_{\cdot \mathrm{OH}}=\ln \left(\frac{[\mathrm{MC}]_{0}}{[\mathrm{MC}]}\right)_{{\mathrm{UV} / \mathrm{H}_{2} \mathrm{O}_{2}}}-\ln \left(\frac{[\mathrm{MC}]_{0}}{[\mathrm{MC}]}\right)_{\mathrm{UV}}=k_{\mathrm{obs}}^{*} \mathrm{t}
$$

However, before the calculation of Equation S12, there is still a point to be considered, which is the shielding effect of $\mathrm{H}_{2} \mathrm{O}_{2}$. Since the control experiments (UV system) do not reflect the UV shielding effect of $\mathrm{H}_{2} \mathrm{O}_{2}$ in $\mathrm{UV} / \mathrm{H}_{2} \mathrm{O}_{2}$ experiments, it is necessary to compensate for the overestimated $\mathrm{MC}$ degradation by multiplying a correction coefficient reflecting actual incident photon flow when $\mathrm{H}_{2} \mathrm{O}_{2}$ is present. Because the photon flow value is exponentially reduced as it passes through the optical pathlength of the photoreactor (Figure S7b), Equation S13 was integrated as a function of the optical pathlength " $x$ " as follows:

$$
\left.\frac{\mathrm{d}[\mathrm{MC}]}{\mathrm{dt}}\right|_{\mathrm{UV}}=-\int_{0}^{\mathrm{b}} I_{\mathrm{x}}\left(10^{-\varepsilon_{\mathrm{MC}}[\mathrm{MC}] \mathrm{dx}}\right) \emptyset_{\mathrm{MC}}=-2.303 \varepsilon_{\mathrm{MC}}[\mathrm{MC}] \emptyset_{\mathrm{MC}} \int_{0}^{\mathrm{b}} I_{\mathrm{x}} \mathrm{dx}
$$

where $I_{\mathrm{x}}$ represents the incident photon flow at the optical pathlength $\mathrm{x} \mathrm{cm}$ in the presence of $\mathrm{H}_{2} \mathrm{O}_{2}\left(I_{x}=I_{0} 10^{-\varepsilon_{H_{2}} \mathrm{O}_{2} x\left[\mathrm{H}_{2} \mathrm{O}_{2}\right]}\right)$. Equation $\mathrm{S} 13$ can be simplified as Equation S14.

$$
\begin{aligned}
& \left.\frac{\mathrm{d}[\mathrm{MC}]}{\mathrm{dt}}\right|_{\mathrm{UV}}=-2.303 \varepsilon_{\mathrm{MC}}[\mathrm{MC}] \emptyset_{\mathrm{MC}} I_{0}\left[\frac{10^{-\varepsilon_{\mathrm{H}_{2} \mathrm{O}_{2}} \times\left[\mathrm{H}_{2} \mathrm{O}_{2}\right]}}{2.303 \varepsilon_{\mathrm{H}_{2} \mathrm{O}_{2}}\left[\mathrm{H}_{2} \mathrm{O}_{2}\right]}\right]_{0}^{\mathrm{b}} \\
& =-2.303 \varepsilon_{\mathrm{MC}}[\mathrm{MC}] \emptyset_{\mathrm{MC}} I_{0}\left(\frac{1-10^{-\mathrm{H}_{2} \mathrm{O}_{2}} \mathrm{~b}\left[\mathrm{H}_{2} \mathrm{O}_{2}\right]}{2.303 \varepsilon_{\mathrm{H}_{2} \mathrm{O}_{2}}\left[\mathrm{H}_{2} \mathrm{O}_{2}\right]}\right) \\
& =-2.303 \varepsilon_{\mathrm{MC}}[\mathrm{MC}] \emptyset_{\mathrm{MC}} I_{0} \mathrm{~b}\left(\frac{1-10^{-\mathrm{E}_{2} \mathrm{O}_{2}} \mathrm{~b}\left[\mathrm{H}_{2} \mathrm{O}_{2}\right]}{2.303 \varepsilon_{\mathrm{H}_{2} \mathrm{O}_{2}} \mathrm{~b}\left[\mathrm{H}_{2} \mathrm{O}_{2}\right]}\right)=-2.303 I_{0} \varepsilon_{\mathrm{MC}} \mathrm{b} \emptyset_{\mathrm{MC}} \alpha[\mathrm{MC}]
\end{aligned}
$$

where $\alpha$ represents the correction coefficient for the control experiment $(\alpha=$ $\left.\frac{1-10^{-\varepsilon} \mathrm{H}_{2} \mathrm{O}_{2} b\left[\mathrm{H}_{2} \mathrm{O}_{2}\right]}{2.303 \varepsilon_{\mathrm{H}_{2} \mathrm{O}_{2}} b\left[\mathrm{H}_{2} \mathrm{O}_{2}\right]}\right)$. Meanwhile, the $\mathrm{b}$ value is not the same as the diameter of the cylindrical 
photo reactor $(2 \mathrm{~cm})$ due to the shape of the photoreactor and the angle of the incident photon (Figure S7c). For this reason, the b value was thereby chemically determined by the photolysis kinetics of $\mathrm{H}_{2} \mathrm{O}_{2}$ using Equations $\mathrm{S} 15$ and $\mathrm{S} 16$.

$$
\begin{aligned}
& \left.\frac{\mathrm{d}\left[\mathrm{H}_{2} \mathrm{O}_{2}\right]}{\mathrm{dt}}\right|_{\mathrm{UV}}=-2.303 I_{0} \varepsilon_{254, \mathrm{H}_{2} \mathrm{O}_{2}} \mathrm{~b} \emptyset_{254, \mathrm{H}_{2} \mathrm{O}_{2}}\left[\mathrm{H}_{2} \mathrm{O}_{2}\right] \\
& \ln \left(\frac{\left[\mathrm{H}_{2} \mathrm{O}_{2}\right]_{0}}{\left[\mathrm{H}_{2} \mathrm{O}_{2}\right]}\right)_{\mathrm{UV}}=2.303 I_{0} \varepsilon_{254, \mathrm{H}_{2} \mathrm{O}_{2}} \mathrm{~b} \emptyset_{254, \mathrm{H}_{2} \mathrm{O}_{2}} \mathrm{t}=k_{\mathrm{obs}, \mathrm{H}_{2} \mathrm{O}_{2}} \mathrm{t}
\end{aligned}
$$

where $\varepsilon_{254, \mathrm{H}_{2} \mathrm{O}_{2}}$ is the molar absorption coefficient of $\mathrm{H}_{2} \mathrm{O}_{2}$ at $254 \mathrm{~nm}\left(19.6 \mathrm{M}^{-1} \mathrm{~cm}^{-1}\right)$, $\emptyset_{254, \mathrm{H}_{2} \mathrm{O}_{2}}$ is the quantum yield of $\mathrm{H}_{2} \mathrm{O}_{2}$ at $254 \mathrm{~nm}\left(0.5 \mathrm{~mol}_{\text {photon }}^{-1} \mathrm{~S} 6\right), k_{o b s, \mathrm{H}_{2} \mathrm{O}_{2}}$ is the observed pseudo-first-order rate constant for the photolysis of $\mathrm{H}_{2} \mathrm{O}_{2}$ and were calculated to be $6.8 \times 10^{-5} \mathrm{~s}^{-1}$ (Figure S9). Using Equation S16, the determined b was $2.37 \mathrm{~cm}$, which was applied into Equation S14. The finally determined $\alpha$ value was 0.94 , and the shielding effect of $\mathrm{H}_{2} \mathrm{O}_{2}$ was corrected by multiplying the term of the UV system in Equation S11 by 0.94 . 


\section{Text S5. Analysis of oxidation products of MCs using liquid chromatography-mass spectrometry (LC/MS)}

The chromatographic separation was carried out using an Acclaim $120 \mathrm{C} 18$ column $(2.1 \times$ $150 \mathrm{~mm}, 5 \mu \mathrm{m}$ ), using mobile phase mixture of $0.1 \%$ formic acid and methanol at a flow rate of $0.3 \mathrm{~mL} \mathrm{~min}^{-1}$. The methanol contents in the mobile phase were $55 \%$ for MC-LR, -RR and YR, and $70 \%$ for MC-LA, -LF and -LW. The heated electrospray ionization source interface was operated in the positive ionization mode under the following conditions: spray voltage, +3.5 kV; sheath gas, 40 arbitrary units; auxiliary gas, 10 arbitrary units; sweep gas, 0 arbitrary units; capillary temperature, $320^{\circ} \mathrm{C}$ and vaporizer temperature, $350^{\circ} \mathrm{C}$. The mass spectra were obtained in full scanning mode from 700 to $1200 \mathrm{~m} / \mathrm{z}$ at a resolution of 35000 , an automatic gain control target value of $5 \times 10^{4}$, and a maximum injection time of $100 \mathrm{~ms}$. For achieving high mass accuracy, external calibration was performed before the analysis. All data acquisition and its processing were performed using Xcalibur 3.0.2 software (Thermo Fisher Scientific Inc., U.S.A.). From the LC/MS data, the products were chosen through the scanning of the peak intensity of total ion current chromatography, and were identified by finding the best match with the exact mass values of the anticipated products (within $5 \mathrm{ppm}$ of mass tolerance). In this study, only monoisotopic mass with hydrogen adduct (i.e., $[\mathrm{M}+\mathrm{H}]^{+}$) was considered. 


\section{Text S6. Analysis of $\mathrm{O}_{3}$ during natural water tests by SFS}

The decrease of $\mathrm{O}_{3}$ during the natural water tests was measured by SFS. The SFS system worked with two $10 \mathrm{~mL}$ syringes, each filled with $\mathrm{O}_{3}$ and natural water samples. The operation conditions of SFS for the natural water experiments were the same as that of Text $\mathrm{S} 2$. The acquisition of the absorbance data at $258 \mathrm{~nm}$ was performed every millisecond until the $\mathrm{O}_{3}$ was completely decomposed. It is important to note that the blank absorbance by the natural water sample should be considered during data processing. To calculate the actual concentration of $\mathrm{O}_{3}$, the absorbance by natural water sample was corrected by the following data fitting process: i) two fit lines are obtained for the two phases (i.e., decreasing and stationary phases) using OriginPro 8.5 software (Figure S10a), ii) after subtracting the two fit lines from each other, the actual $\mathrm{O}_{3}$ concentrations were calculated using the molar absorption coefficients of $\mathrm{O}_{3}$ (Figure $\mathrm{S} 10 \mathrm{~b}$ ). The variations in DOM absorbance after ozonation (by comparing the absorbance before and after the ozone treatment) were less than $5 \%$ of those of $\mathrm{O}_{3}$ absorbance in all experiments, indicating that the effect of DOM on these measurements was negligible. 
Table S1. Summary of $k_{\mathrm{O} 3, \mathrm{CA}}$ values at different $\mathrm{pH}$ values (temperature $=20 \pm 1^{\circ} \mathrm{C}$ )

\begin{tabular}{|c|c|c|c|}
\hline $\mathrm{pH}$ & $\mathrm{O}_{3}(\mu \mathrm{M})$ & $k_{\mathrm{obs}}\left(\mathrm{s}^{-1}\right)$ & $k_{\mathrm{O} 3, \mathrm{CA}}\left(\mathrm{M}^{-1} \mathrm{~s}^{-1}\right)$ \\
\hline \multirow{4}{*}{2.3} & 47.5 & $2.57 \pm 0.07$ & \multirow{4}{*}{$5.84 \pm 0.04 \times 10^{4}$} \\
\hline & 65.2 & $3.76 \pm 0.09$ & \\
\hline & 76.5 & $4.58 \pm 0.05$ & \\
\hline & 96.5 & $5.44 \pm 0.03$ & \\
\hline \multirow{4}{*}{3.6} & 46.5 & $4.98 \pm 0.05$ & \multirow{4}{*}{$1.16 \pm 0.01 \times 10^{5}$} \\
\hline & 65.1 & $7.63 \pm 0.13$ & \\
\hline & 78.8 & $9.40 \pm 0.10$ & \\
\hline & 94.2 & $10.9 \pm 0.08$ & \\
\hline \multirow{4}{*}{4.5} & 43 & $13.0 \pm 0.25$ & \multirow{4}{*}{$3.26 \pm 0.12 \times 10^{5}$} \\
\hline & 53.4 & $17.3 \pm 1.09$ & \\
\hline & 70.0 & $22.9 \pm 0.79$ & \\
\hline & 81.4 & $27.2 \pm 1.25$ & \\
\hline \multirow{4}{*}{5.3} & 44.4 & $22.1 \pm 0.39$ & \multirow{4}{*}{$4.98 \pm 0.14 \times 10^{5}$} \\
\hline & 53.4 & $26.9 \pm 0.54$ & \\
\hline & 57.5 & $29.1 \pm 0.67$ & \\
\hline & 81.4 & $39.9 \pm 1.97$ & \\
\hline \multirow{4}{*}{6.3} & 32.3 & $23.3 \pm 0.31$ & \multirow{4}{*}{$7.47 \pm 0.01 \times 10^{5}$} \\
\hline & 45.5 & $30.6 \pm 1.26$ & \\
\hline & 53.6 & $40.3 \pm 0.13$ & \\
\hline & 60.9 & $46.4 \pm 0.38$ & \\
\hline \multirow{8}{*}{7.2} & $28.7 \pm 2.80$ & $20.8 \pm 0.22$ & \multirow{8}{*}{$7.78 \pm 0.07 \times 10^{5}$} \\
\hline & $40.9 \pm 1.53$ & $30.1 \pm 1.02$ & \\
\hline & 47.7 & $36.7 \pm 0.76$ & \\
\hline & $69.6 \pm 0.54$ & $55.0 \pm 1.23$ & \\
\hline & 55.6 & $42.1 \pm 0.73$ & \\
\hline & 66.3 & $49.6 \pm 0.88$ & \\
\hline & 86.5 & $68.6 \pm 2.95$ & \\
\hline & 106.4 & $84.3 \pm 0.32$ & \\
\hline
\end{tabular}




\begin{tabular}{llll}
\hline & 34.8 & $27.3 \pm 0.5$ & \\
44.8 & $34.9 \pm 1.15$ & \\
45.4 & $34.9 \pm 1.13$ & \\
& 54.8 & $41.6 \pm 2.21$ & $7.72 \pm 0.08 \times 10^{5}$ \\
& 59.1 & $45.3 \pm 1.13$ & \\
67.1 & $52.2 \pm 1.66$ & \\
& 72.3 & $55.8 \pm 1.19$ & \\
& 35.1 & $26.5 \pm 0.69$ & \\
& 41.3 & $32.9 \pm 2.46$ & \\
& 48.5 & $41.1 \pm 1.52$ & \\
& 59.7 & $47.6 \pm 0.94$ & \\
& & & \\
\hline
\end{tabular}


Table S2. Summary of $k_{\mathrm{O} 3, \mathrm{CA}}$ values at different temperatures $(\mathrm{pH}=7.2)$

\begin{tabular}{|c|c|c|c|}
\hline Temperature $\left({ }^{\circ} \mathrm{C}\right)$ & $\mathrm{O}_{3}(\mu \mathrm{M})$ & $k_{\mathrm{obs}}\left(\mathrm{s}^{-1}\right)$ & $k_{\mathrm{O} 3, \mathrm{CA}}\left(\mathrm{M}^{-1} \mathrm{~s}^{-1}\right)$ \\
\hline \multirow{4}{*}{7.5} & 441.8 & $22.6 \pm 0.53$ & \multirow{4}{*}{$5.44 \pm 0.11 \times 10^{5}$} \\
\hline & 60.9 & $32.9 \pm 0.79$ & \\
\hline & 71.1 & $38.3 \pm 0.93$ & \\
\hline & 99.8 & $54.8 \pm 1.62$ & \\
\hline \multirow{4}{*}{10} & 34.1 & $18.4 \pm 0.07$ & \multirow{4}{*}{$5.83 \pm 0.14 \times 10^{5}$} \\
\hline & 46.1 & $26.2 \pm 0.35$ & \\
\hline & 60.5 & $34.3 \pm 0.95$ & \\
\hline & 69.5 & $42.5 \pm 1.67$ & \\
\hline \multirow{4}{*}{12.5} & 59.8 & $33.7 \pm 2.87$ & \multirow{4}{*}{$6.31 \pm 0.19 \times 10^{5}$} \\
\hline & 82.5 & $51.5 \pm 0.79$ & \\
\hline & 98.1 & $62.4 \pm 2.38$ & \\
\hline & 116.4 & $75.6 \pm 4.63$ & \\
\hline \multirow{4}{*}{15} & 34.5 & $20.9 \pm 0.52$ & \multirow{4}{*}{$6.71 \pm 0.09 \times 10^{5}$} \\
\hline & 48.9 & $31.9 \pm 0.33$ & \\
\hline & 51.2 & $34.1 \pm 1.00$ & \\
\hline & 65.1 & $44.9 \pm 1.89$ & \\
\hline \multirow{8}{*}{20} & $28.7 \pm 2.80$ & $20.8 \pm 0.22$ & \multirow{8}{*}{$7.78 \pm 0.07 \times 10^{5}$} \\
\hline & $40.9 \pm 1.53$ & $30.1 \pm 1.02$ & \\
\hline & 47.7 & $36.7 \pm 0.76$ & \\
\hline & $69.6 \pm 0.54$ & $55.0 \pm 1.23$ & \\
\hline & 55.6 & $42.1 \pm 0.73$ & \\
\hline & 66.3 & $49.6 \pm 0.88$ & \\
\hline & 86.5 & $68.6 \pm 2.95$ & \\
\hline & 106.4 & $84.3 \pm 0.32$ & \\
\hline \multirow{4}{*}{25} & 30 & $26.5 \pm 1.52$ & \multirow{4}{*}{$8.77 \pm 0.25 \times 10^{5}$} \\
\hline & 37.5 & $26.2 \pm 0.73$ & \\
\hline & 38.3 & $35.1 \pm 2.79$ & \\
\hline & 85 & $73.8 \pm 3.89$ & \\
\hline
\end{tabular}


Table S3. Characterization of the concentrated natural waters after dilution in half with deionized water

\begin{tabular}{ccc}
\hline \multirow{2}{*}{ Parameters } & \multicolumn{2}{c}{ Natural waters } \\
\cline { 2 - 3 } & Maegok & Gamak \\
\hline \hline DOC $\left(\mathrm{mgC} \mathrm{L}^{-1}\right)$ & $5.06 \pm 0.12$ & $2.66 \pm 0.06$ \\
$\mathrm{UV}_{254}$ & 0.105 & 0.046 \\
$\mathrm{SUVA}_{254}\left(\mathrm{~L} \mathrm{mg} \mathrm{C}^{-1} \mathrm{~m}^{-1}\right)$ & $2.07 \pm 0.07$ & $1.73 \pm 0.08$ \\
$\mathrm{pH}$ & 7.65 & 7.95 \\
\hline
\end{tabular}


Table S4. $\int\left[\mathrm{O}_{3}\right] \mathrm{dt}$ in Maegok and Gamak natural waters at various $\mathrm{O}_{3}$ doses

\begin{tabular}{|c|c|c|c|}
\hline \multicolumn{2}{|c|}{ Maegok } & \multicolumn{2}{|c|}{ Gamak } \\
\hline $\mathrm{O}_{3}(\mathrm{M})$ & $\int\left[\mathrm{O}_{3}\right] \mathrm{dt}(\mathrm{M} \mathrm{s})$ & $\mathrm{O}_{3}(\mathrm{M})$ & $\int\left[\mathrm{O}_{3}\right] \mathrm{dt}(\mathrm{M} \mathrm{s})$ \\
\hline $4.86 \times 10^{-6}$ & $3.07 \times 10^{-7}$ & $2.33 \times 10^{-6}$ & $2.00 \times 10^{-7}$ \\
\hline $4.27 \times 10^{-6}$ & $2.94 \times 10^{-7}$ & $2.27 \times 10^{-6}$ & $1.30 \times 10^{-7}$ \\
\hline $4.39 \times 10^{-6}$ & $3.97 \times 10^{-7}$ & $1.93 \times 10^{-6}$ & $1.33 \times 10^{-7}$ \\
\hline $8.06 \times 10^{-6}$ & $1.11 \times 10^{-6}$ & $3.39 \times 10^{-6}$ & $2.71 \times 10^{-7}$ \\
\hline $7.37 \times 10^{-6}$ & $9.21 \times 10^{-7}$ & $3.59 \times 10^{-6}$ & $2.30 \times 10^{-7}$ \\
\hline $7.07 \times 10^{-6}$ & $8.00 \times 10^{-7}$ & $3.38 \times 10^{-6}$ & $1.87 \times 10^{-7}$ \\
\hline $9.79 \times 10^{-6}$ & $1.62 \times 10^{-6}$ & $6.45 \times 10^{-6}$ & $1.62 \times 10^{-6}$ \\
\hline $8.42 \times 10^{-6}$ & $1.17 \times 10^{-6}$ & $5.91 \times 10^{-6}$ & $9.48 \times 10^{-7}$ \\
\hline $8.66 \times 10^{-6}$ & $1.20 \times 10^{-6}$ & $4.42 \times 10^{-6}$ & $8.66 \times 10^{-7}$ \\
\hline $1.23 \times 10^{-5}$ & $2.83 \times 10^{-6}$ & $6.90 \times 10^{-6}$ & $1.60 \times 10^{-6}$ \\
\hline $1.17 \times 10^{-5}$ & $2.74 \times 10^{-6}$ & $6.02 \times 10^{-6}$ & $1.45 \times 10^{-6}$ \\
\hline $1.13 \times 10^{-5}$ & $2.42 \times 10^{-6}$ & $5.45 \times 10^{-6}$ & $1.17 \times 10^{-6}$ \\
\hline $1.26 \times 10^{-5}$ & $3.03 \times 10^{-6}$ & $8.01 \times 10^{-6}$ & $2.49 \times 10^{-6}$ \\
\hline $1.26 \times 10^{-5}$ & $3.26 \times 10^{-6}$ & $7.82 \times 10^{-6}$ & $2.18 \times 10^{-6}$ \\
\hline \multirow[t]{10}{*}{$1.20 \times 10^{-5}$} & $3.05 \times 10^{-6}$ & $8.08 \times 10^{-6}$ & $2.13 \times 10^{-6}$ \\
\hline & & $8.79 \times 10^{-6}$ & $3.33 \times 10^{-6}$ \\
\hline & & $8.97 \times 10^{-6}$ & $2.99 \times 10^{-6}$ \\
\hline & & $8.70 \times 10^{-6}$ & $3.03 \times 10^{-6}$ \\
\hline & & $1.32 \times 10^{-5}$ & $7.61 \times 10^{-6}$ \\
\hline & & $1.27 \times 10^{-5}$ & $6.81 \times 10^{-6}$ \\
\hline & & $1.23 \times 10^{-5}$ & $6.47 \times 10^{-6}$ \\
\hline & & $1.49 \times 10^{-5}$ & $1.44 \times 10^{-5}$ \\
\hline & & $1.36 \times 10^{-5}$ & $1.26 \times 10^{-5}$ \\
\hline & & $1.46 \times 10^{-5}$ & $1.20 \times 10^{-5}$ \\
\hline
\end{tabular}


Table S5. $\int\left[\bullet^{\bullet} \mathrm{OH}\right] \mathrm{dt}$ in Maegok and Gamak natural waters at various $\mathrm{O}_{3}$ dose

\begin{tabular}{cc|cc}
\hline \multicolumn{2}{c|}{ Maegok } & \multicolumn{2}{c}{ Gamak } \\
$\mathrm{O}_{3}(\mathrm{M})$ & $\int\left[{ }^{\circ} \mathrm{OH}\right] \mathrm{dt}(\mathrm{M} \mathrm{s})$ & $\mathrm{O}_{3}(\mathrm{M})$ & $\int\left[{ }^{\bullet} \mathrm{OH}\right] \mathrm{dt}(\mathrm{M} \mathrm{s})$ \\
\hline \hline $2.08 \times 10^{-6}$ & $5.08 \pm 2.92 \times 10^{-12}$ & $2.08 \times 10^{-6}$ & $7.79 \pm 6.09 \times 10^{-12}$ \\
$4.17 \times 10^{-6}$ & $9.88 \pm 7.67 \times 10^{-12}$ & $4.17 \times 10^{-6}$ & $1.52 \pm 0.77 \times 10^{-11}$ \\
$6.25 \times 10^{-6}$ & $1.59 \pm 0.69 \times 10^{-11}$ & $6.25 \times 10^{-6}$ & $2.56 \pm 0.47 \times 10^{-11}$ \\
$8.33 \times 10^{-6}$ & $2.32 \pm 0.07 \times 10^{-11}$ & $8.33 \times 10^{-6}$ & $3.57 \times 10^{-11}$ \\
$1.04 \times 10^{-5}$ & $3.04 \pm 0.32 \times 10^{-11}$ & $1.04 \times 10^{-5}$ & $4.50 \pm 0.41 \times 10^{-11}$ \\
$1.25 \times 10^{-5}$ & $3.54 \pm 0.29 \times 10^{-11}$ & $1.25 \times 10^{-5}$ & $5.34 \pm 0.53 \times 10^{-11}$ \\
\hline
\end{tabular}


Table S6. Oxidation products of MCs identified by LC/MS

\begin{tabular}{|c|c|c|c|c|}
\hline Products & $\begin{array}{l}\text { Chemical formula } \\
\qquad[\mathrm{M}+\mathrm{H}]^{+}\end{array}$ & $\begin{array}{c}\text { Theoretical } \\
\mathrm{m} / \mathrm{z}\end{array}$ & $\begin{array}{c}\triangle^{\mathrm{a}} \\
(\mathrm{ppm})\end{array}$ & $\begin{array}{c}\text { RT } \\
(\min )\end{array}$ \\
\hline MC-LR & $\mathrm{C}_{49} \mathrm{H}_{75} \mathrm{~N}_{10} \mathrm{O}_{12}$ & 995.5560 & 1.51 & 23.6 \\
\hline PLR1 & $\mathrm{C}_{34} \mathrm{H}_{55} \mathrm{~N}_{10} \mathrm{O}_{12}$ & 795.3995 & -0.25 & 1.8 \\
\hline PLR2 & $\mathrm{C}_{37} \mathrm{H}_{59} \mathrm{~N}_{10} \mathrm{O}_{12}$ & 835.4308 & 0.24 & 1.96 \\
\hline MC-RR & $\mathrm{C}_{49} \mathrm{H}_{76} \mathrm{~N}_{13} \mathrm{O}_{12}$ & 1038.5730 & 0.57 & 9.46 \\
\hline PRR1 & $\mathrm{C}_{34} \mathrm{H}_{56} \mathrm{~N}_{13} \mathrm{O}_{12}$ & 838.4165 & 0.24 & 1.96 \\
\hline PRR2 & $\mathrm{C}_{37} \mathrm{H}_{60} \mathrm{~N}_{13} \mathrm{O}_{12}$ & 878.4478 & -0.25 & 1.8 \\
\hline MC-LA & $\mathrm{C}_{46} \mathrm{H}_{68} \mathrm{~N}_{7} \mathrm{O}_{12}$ & 910.4920 & -0.22 & 5.46 \\
\hline PLA1 & $\mathrm{C}_{31} \mathrm{H}_{48} \mathrm{~N}_{7} \mathrm{O}_{12}$ & 710.3355 & -1.26 & 1.76 \\
\hline PLA2 & $\mathrm{C}_{34} \mathrm{H}_{52} \mathrm{~N}_{7} \mathrm{O}_{12}$ & 750.3668 & -1.19 & 1.8 \\
\hline MC-LF & $\mathrm{C}_{52} \mathrm{H}_{72} \mathrm{~N}_{7} \mathrm{O}_{12}$ & 986.5233 & 2.83 & 11.57 \\
\hline PLF1 & $\mathrm{C}_{37} \mathrm{H}_{52} \mathrm{~N}_{7} \mathrm{O}_{12}$ & 786.3668 & -1.14 & 2.32 \\
\hline PLF2 & $\mathrm{C}_{40} \mathrm{H}_{56} \mathrm{~N}_{7} \mathrm{O}_{12}$ & 826.3981 & 0.24 & 2.29 \\
\hline MC-YR & $\mathrm{C}_{52} \mathrm{H}_{73} \mathrm{~N}_{10} \mathrm{O}_{13}$ & 1045.5353 & 1.24 & 18.94 \\
\hline PYR1 & $\mathrm{C}_{37} \mathrm{H}_{51} \mathrm{~N}_{10} \mathrm{O}_{14}$ & 859.3580 & 0.35 & 1.54 \\
\hline PYR2 & $\mathrm{C}_{37} \mathrm{H}_{53} \mathrm{~N}_{10} \mathrm{O}_{14}$ & 861.3737 & -0.58 & 1.56 \\
\hline PYR3 & $\mathrm{C}_{40} \mathrm{H}_{55} \mathrm{~N}_{10} \mathrm{O}_{14}$ & 899.3893 & 1.45 & 1.62 \\
\hline PYR4 & $\mathrm{C}_{40} \mathrm{H}_{57} \mathrm{~N}_{10} \mathrm{O}_{14}$ & 901.4050 & -0.55 & 1.59 \\
\hline PYR5 & $\mathrm{C}_{52} \mathrm{H}_{71} \mathrm{~N}_{10} \mathrm{O}_{14}$ & 1059.5145 & 1.23 & 11.99 \\
\hline PYR6 & $\mathrm{C}_{52} \mathrm{H}_{73} \mathrm{~N}_{10} \mathrm{O}_{14}$ & 1061.5302 & 0.94 & $15.03,16.04$ \\
\hline PYR7 & $\mathrm{C}_{52} \mathrm{H}_{73} \mathrm{~N}_{10} \mathrm{O}_{15}$ & 1077.5251 & 0.56 & 14.57 \\
\hline MC-LW & $\mathrm{C}_{54} \mathrm{H}_{73} \mathrm{~N}_{8} \mathrm{O}_{12}$ & 1025.5342 & -0.98 & 8.94 \\
\hline PLW1 & $\mathrm{C}_{38} \mathrm{H}_{53} \mathrm{~N}_{8} \mathrm{O}_{14}$ & 845.3675 & 1.42 & 1.81 \\
\hline PLW2 & $\mathrm{C}_{39} \mathrm{H}_{53} \mathrm{~N}_{8} \mathrm{O}_{15}$ & 873.3624 & -1.95 & 1.88 \\
\hline PLW3 & $\mathrm{C}_{41} \mathrm{H}_{57} \mathrm{~N}_{8} \mathrm{O}_{14}$ & 885.3988 & 3.39 & 1.97 \\
\hline PLW4 & $\mathrm{C}_{53} \mathrm{H}_{73} \mathrm{~N}_{8} \mathrm{O}_{13}$ & 1029.5291 & 0.87 & 6.33 \\
\hline PLW5 & $\mathrm{C}_{53} \mathrm{H}_{73} \mathrm{~N}_{8} \mathrm{O}_{14}$ & 1045.5240 & 0.96 & 6.05 \\
\hline PLW6 & $\mathrm{C}_{54} \mathrm{H}_{73} \mathrm{~N}_{8} \mathrm{O}_{14}$ & 1057.5240 & 1.13 & 6.45 \\
\hline PLW7 & $\mathrm{C}_{54} \mathrm{H}_{73} \mathrm{~N}_{8} \mathrm{O}_{15}$ & 1073.5189 & 1.02 & $3.76,4.72,5.06$ \\
\hline
\end{tabular}

${ }^{\mathrm{a}} \triangle$ represents the mass tolerance (maximal difference between an experimental mass and a theoretical mass, ppm). 
Table S7. Specific $\mathrm{O}_{3}$ doses required for $99 \%$ removal of MCs in Maegok and Gamak natural waters.

\begin{tabular}{ccc}
\hline MCs & $\begin{array}{r}\text { Specific } \mathrm{O}_{3} \text { dose for 99\% MC removal }\left(\mathrm{g} \mathrm{O}_{3} \mathrm{~g} \mathrm{DOC}^{-1}\right) \\
\text { Maegok }\end{array}$ & $\begin{array}{c}\text { Gamak } \\
\text { LW }\end{array}$ \\
\hline YR & 0.08 & 0.06 \\
RR & 0.12 & 0.10 \\
LR & 0.17 & 0.14 \\
LA & 0.17 & 0.14 \\
LF & 0.17 & 0.14 \\
\hline
\end{tabular}

*All values were calculated based on the results of Figure 2. 

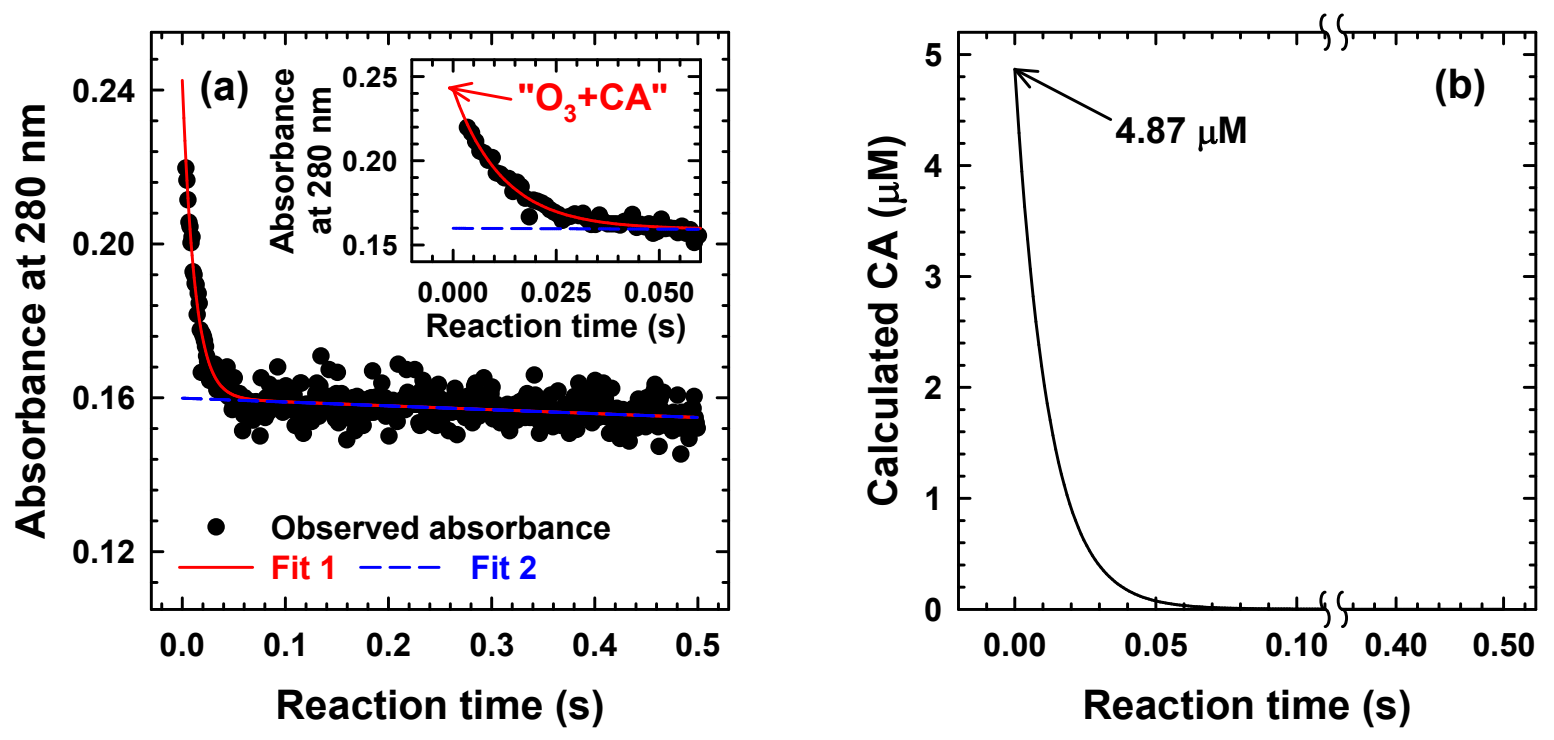

Figure S1. Example of measurement of CA concentration. (a) Absorbance at $280 \mathrm{~nm}$ during CA oxidation by excess $\mathrm{O}_{3}$. Red and bule dotted lines represent fits of the absorbance regarding whole examined reaction time and stationary phases (from 0.2 to $0.5 \mathrm{~s}$ ), respectively. (b) Calculated CA from the subtraction of blue dotted line from red line in (a) $\left([\mathrm{CA}]_{0}=5 \mu \mathrm{M},\left[\mathrm{O}_{3}\right]_{0}=83.1 \mu \mathrm{M},[t-\mathrm{BuOH}]_{0}=5 \mathrm{mM}, \mathrm{pH}=7.2\right.$, temperature $\left.=20 \pm 1^{\circ} \mathrm{C}\right)$. 


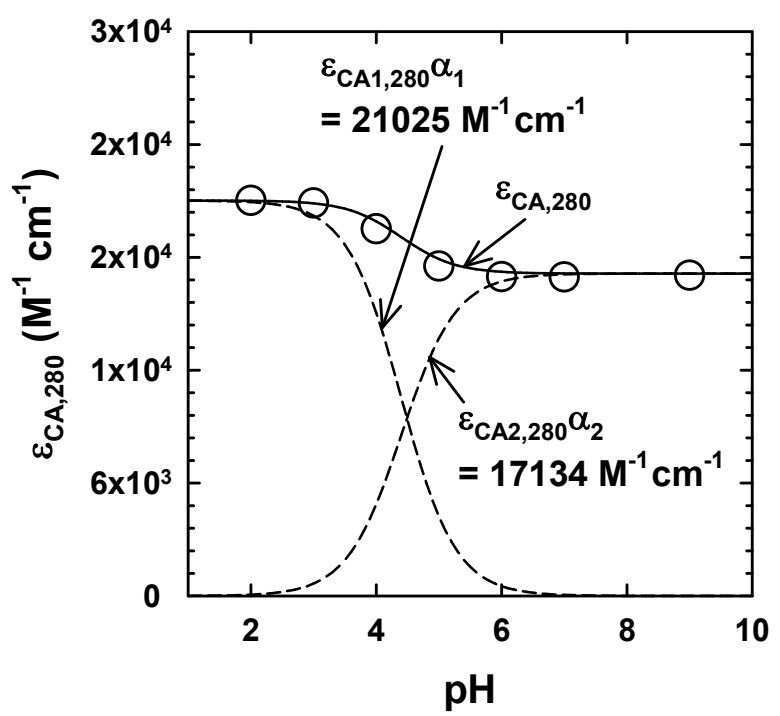

Figure S2. pH-dependent molar absorption coefficient of CA at $280 \mathrm{~nm}$. 

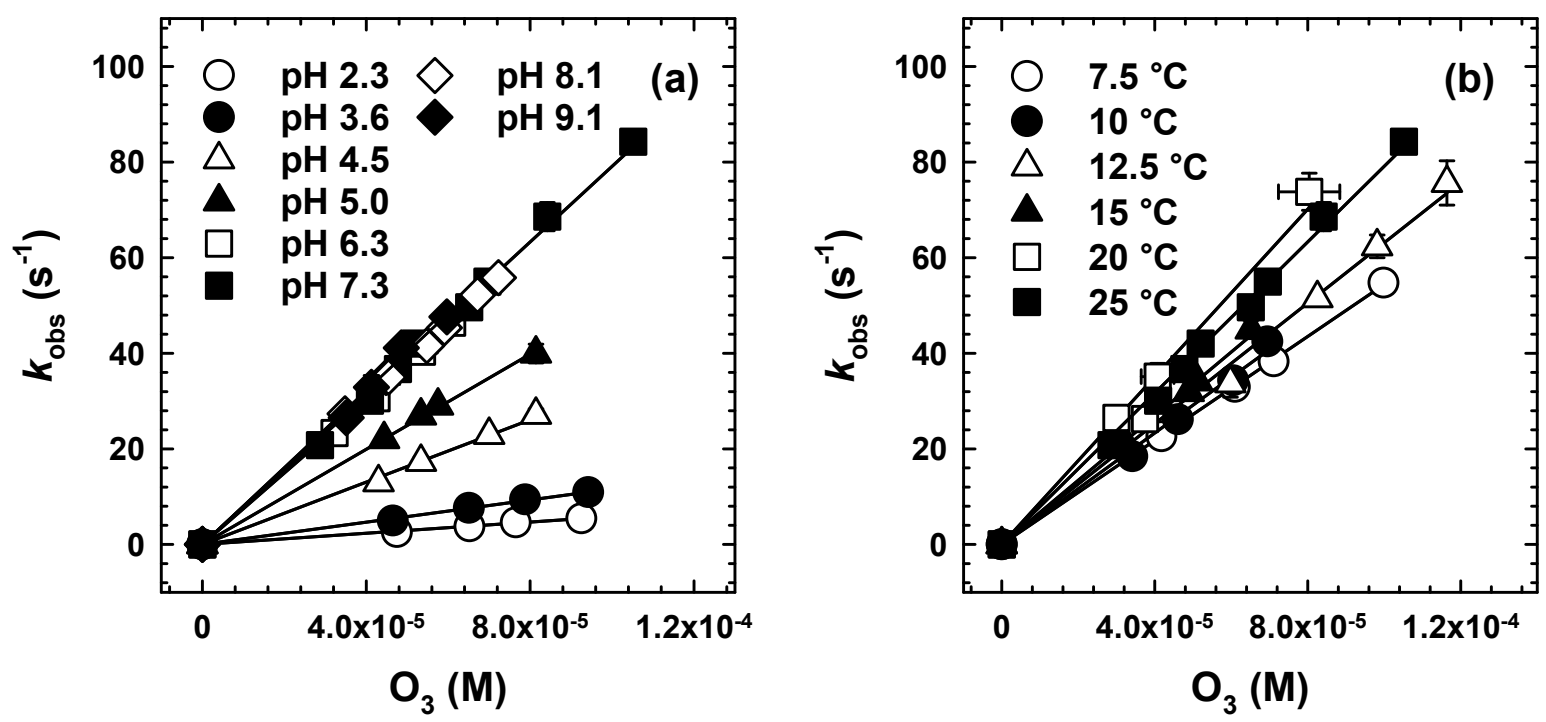

Figure S3. Plot of pseudo-first-order rate constants of CA decomposition $\left(k_{\mathrm{obs}}\right)$ as a function of $\mathrm{O}_{3}$ concentration at different (a) $\mathrm{pH}$ values and (b) temperatures $\left([\mathrm{CA}]_{0}=5 \mu \mathrm{M},[t-\mathrm{BuOH}]_{0}\right.$ $=5 \mathrm{mM}$, temperature $=20 \pm 1^{\circ} \mathrm{C}$ for $(\mathrm{a})$ and (b); temperature $=20 \pm 1^{\circ} \mathrm{C}$ for (a), $\mathrm{pH}=7.2$ for (b)). 

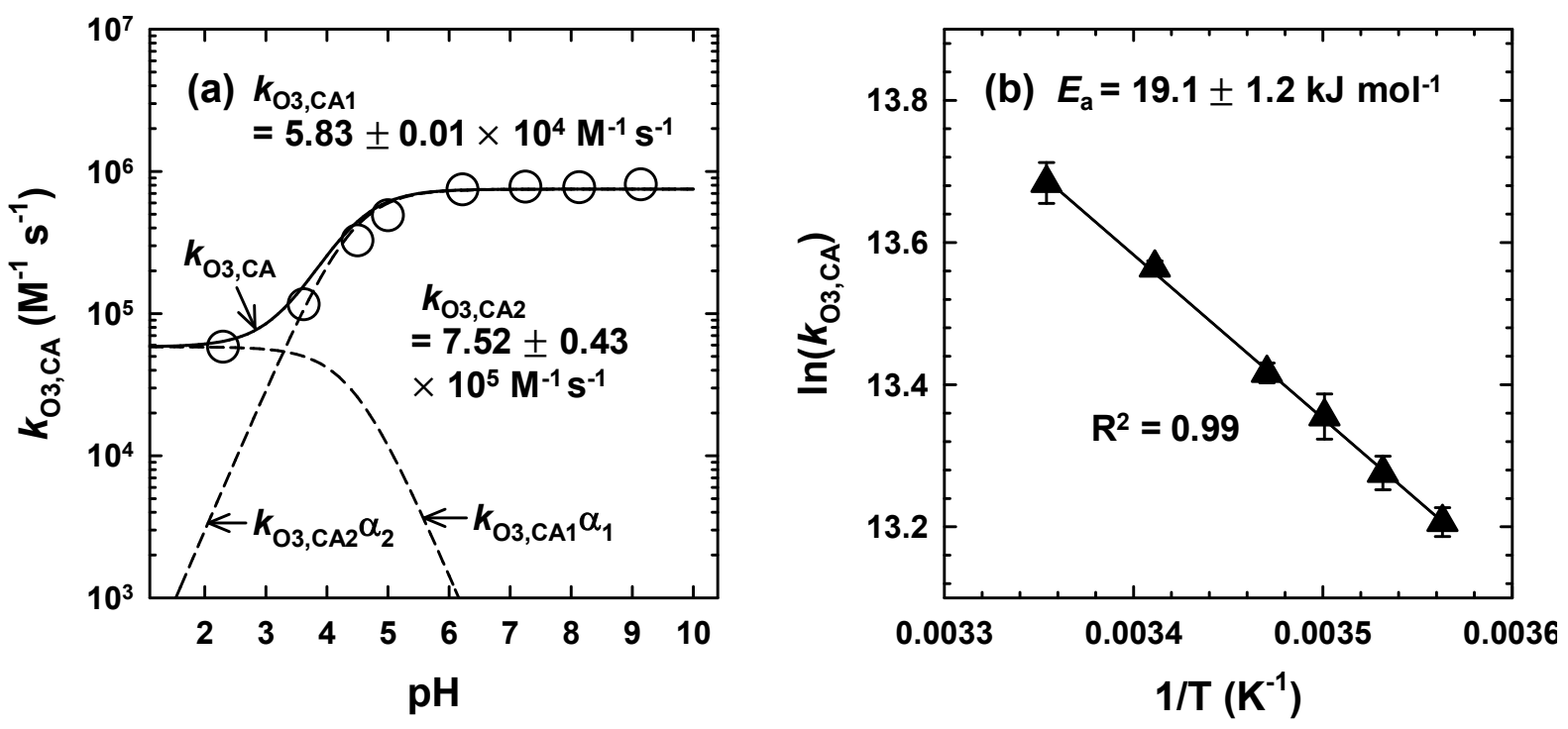

Figure S4. (a) pH-dependence of $k_{\mathrm{O} 3, \mathrm{CA}}$, and (b) Arrhenius plot of $k_{\mathrm{O} 3, \mathrm{CA}}$. Symbols and lines in (a) represent experimental data, model prediction of $k_{\mathrm{O} 3, \mathrm{CA}}$ (solid) and $k_{\mathrm{O} 3, \mathrm{CA} i} \alpha_{i}$ (dashed), respectively $\left([\mathrm{CA}]_{0}=5 \mu \mathrm{M},\left[\mathrm{O}_{3}\right]_{0}=28.7-118.4 \mu \mathrm{M},[t-\mathrm{BuOH}]_{0}=5 \mathrm{mM}\right.$ for (a) and (b); temperature $=20 \pm 1{ }^{\circ} \mathrm{C}$ for $(\mathrm{a}), \mathrm{pH}=7.2$ for $\left.(\mathrm{b})\right)$. 


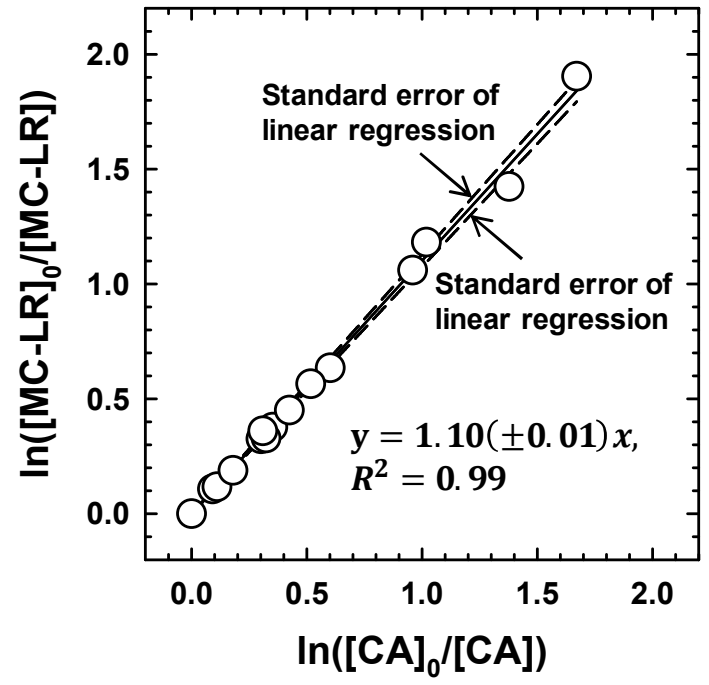

Figure S5. Example of CK plot for the determination of $k_{\mathrm{O}, \mathrm{MC}-\mathrm{LR}}$ ([MC-LR $]_{0}=0.1 \mu \mathrm{M}$, $[\mathrm{CA}]_{0}=0.1 \mu \mathrm{M},\left[\mathrm{O}_{3}\right]_{0}=0.02-0.2 \mu \mathrm{M},[t-\mathrm{BuOH}]_{0}=5 \mathrm{mM}, \mathrm{pH}=7.2$, temperature $=20 \pm 1^{\circ} \mathrm{C}$ ) 

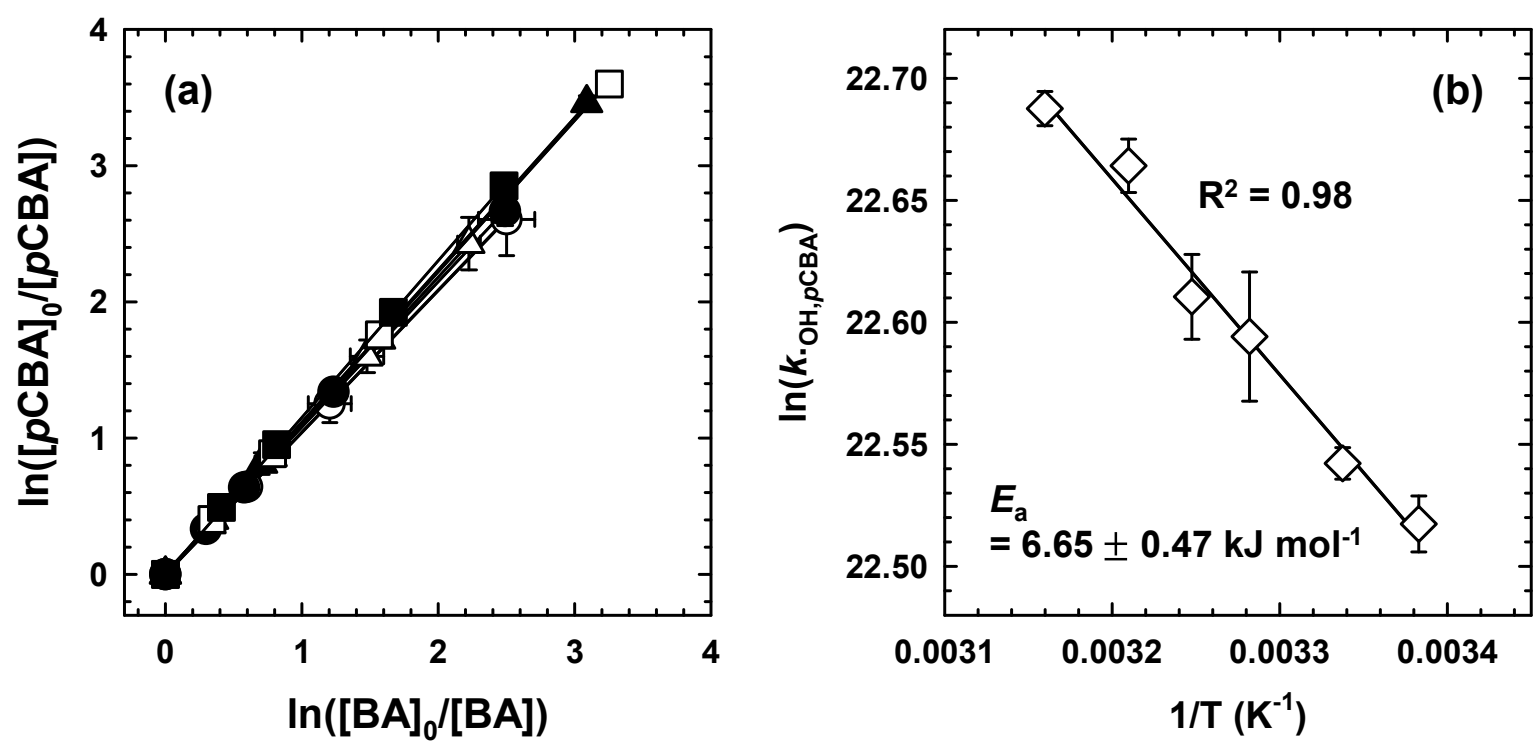
$22.3^{\circ} \mathrm{C}, \mathrm{y}=1.04 \mathrm{x}, \mathrm{k}_{\text {. } \mathrm{OH}, \mathrm{pCBA}}=6.01 \times 10^{9} \mathrm{M}^{-1} \mathrm{~s}^{-1}$
$26.4^{\circ} \mathrm{C}, \mathrm{y}=1.08 \mathrm{x}, k_{\text {. } \mathrm{OH}, \mathrm{pCBA}}=6.17 \times 10^{9} \mathrm{M}^{-1} \mathrm{~s}^{-1}$
$\triangle 31.4^{\circ} \mathrm{C}, \mathrm{y}=1.09 \mathrm{x}, k_{\text {- } \mathrm{OH}, \mathrm{pCBA}}=6.50 \times 10^{9} \mathrm{M}^{-1} \mathrm{~s}^{-1}$
$\triangle 34.7^{\circ} \mathrm{C}, \mathrm{y}=1.12 \mathrm{x}, \mathrm{k}_{\text {. } \mathrm{OH}, \mathrm{pCBA}}=6.60 \times 10^{9} \mathrm{M}^{-1} \mathrm{~s}^{-1}$
$\square \quad 38.5^{\circ} \mathrm{C}, \mathrm{y}=1.11 \mathrm{x}, k_{\cdot \mathrm{OH}, p \mathrm{PBA}}=6.97 \times 10^{9} \mathrm{M}^{-1} \mathrm{~s}^{-1}$
- $43.4^{\circ} \mathrm{C}, \mathrm{y}=1.15 \mathrm{x}, \mathrm{k}_{\text {-OH }, p \mathrm{PCB}}=7.13 \times 10^{9} \mathrm{M}^{-1} \mathrm{~s}^{-1}$

Figure S6. (a) CK plot for the determination of $k_{\cdot 0 \mathrm{OH}, p \mathrm{CBA}}$ at different temperatures, and (b) Arrhenius plot of $k_{\bullet \circ} \mathrm{OH}, p \mathrm{CBA}\left([\mathrm{BA}]_{0}=0.1 \mu \mathrm{M},[p \mathrm{CBA}]_{0}=0.1 \mu \mathrm{M},\left[\mathrm{H}_{2} \mathrm{O}_{2}\right]_{0}=10 \mathrm{mM}, \mathrm{pH}=\right.$ 7.5). 


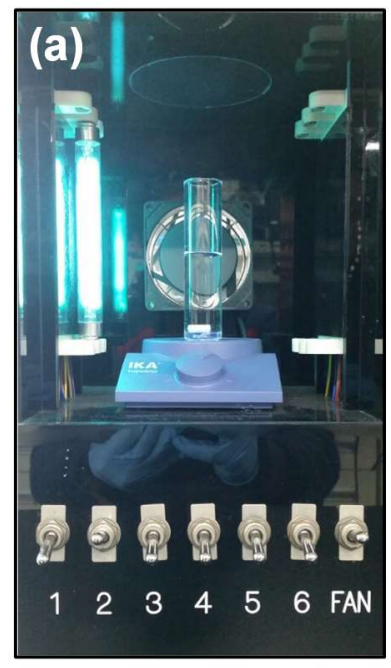

(b)

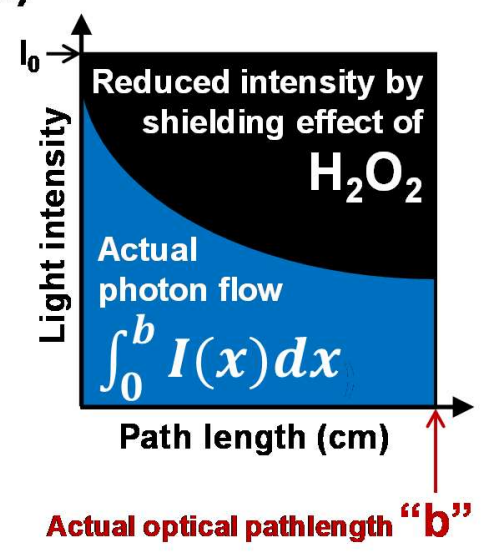

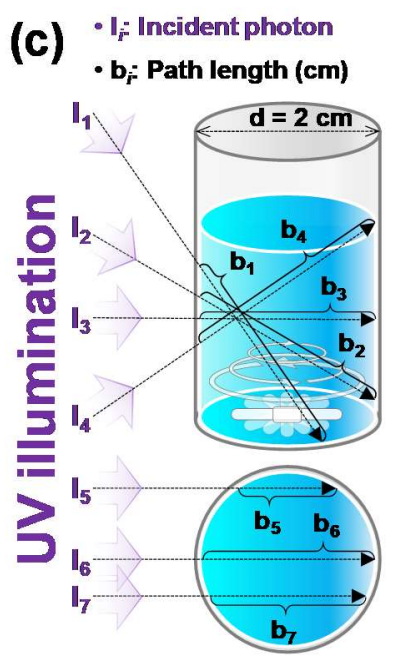

Figure S7. (a) Quartz photoreactor with UV illumination, (b) actual photon flow considered by shielding effect of $\mathrm{H}_{2} \mathrm{O}_{2}$ and the acutual optical pathlength, and (c) the optical pathlength variation by different incidence angle of photon and reactor shape. 


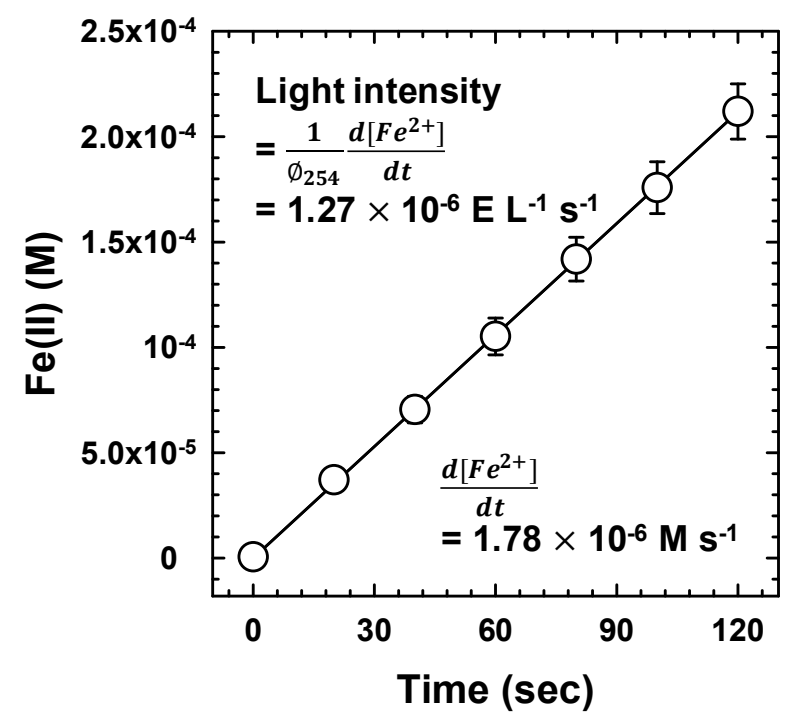

Figure S8. Reduction of ferrioxalate by UV as a function of illumination time $\left([\mathrm{Fe}(\mathrm{III})]_{0}=\right.$

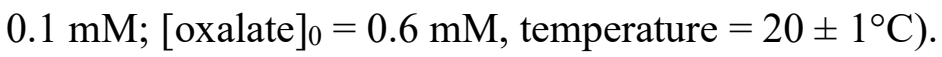




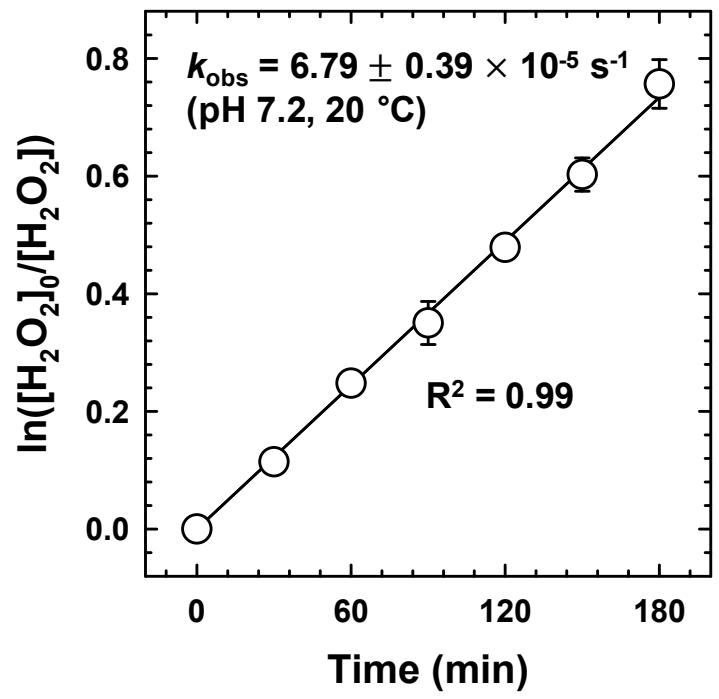

Figure S9. Time-dependent profile of $\ln \left(\left[\mathrm{H}_{2} \mathrm{O}_{2}\right]_{0} /\left[\mathrm{H}_{2} \mathrm{O}_{2}\right]\right)$ during UV illumination $\left(\left[\mathrm{H}_{2} \mathrm{O}_{2}\right]_{0}=\right.$ $0.1 \mathrm{mM},[t-\mathrm{BuOH}]_{0}=10 \mathrm{mM},[\mathrm{PBS}]=10 \mathrm{mM}, \mathrm{pH}=7.2$, temperature $=21 \pm 1^{\circ} \mathrm{C}$ ). 

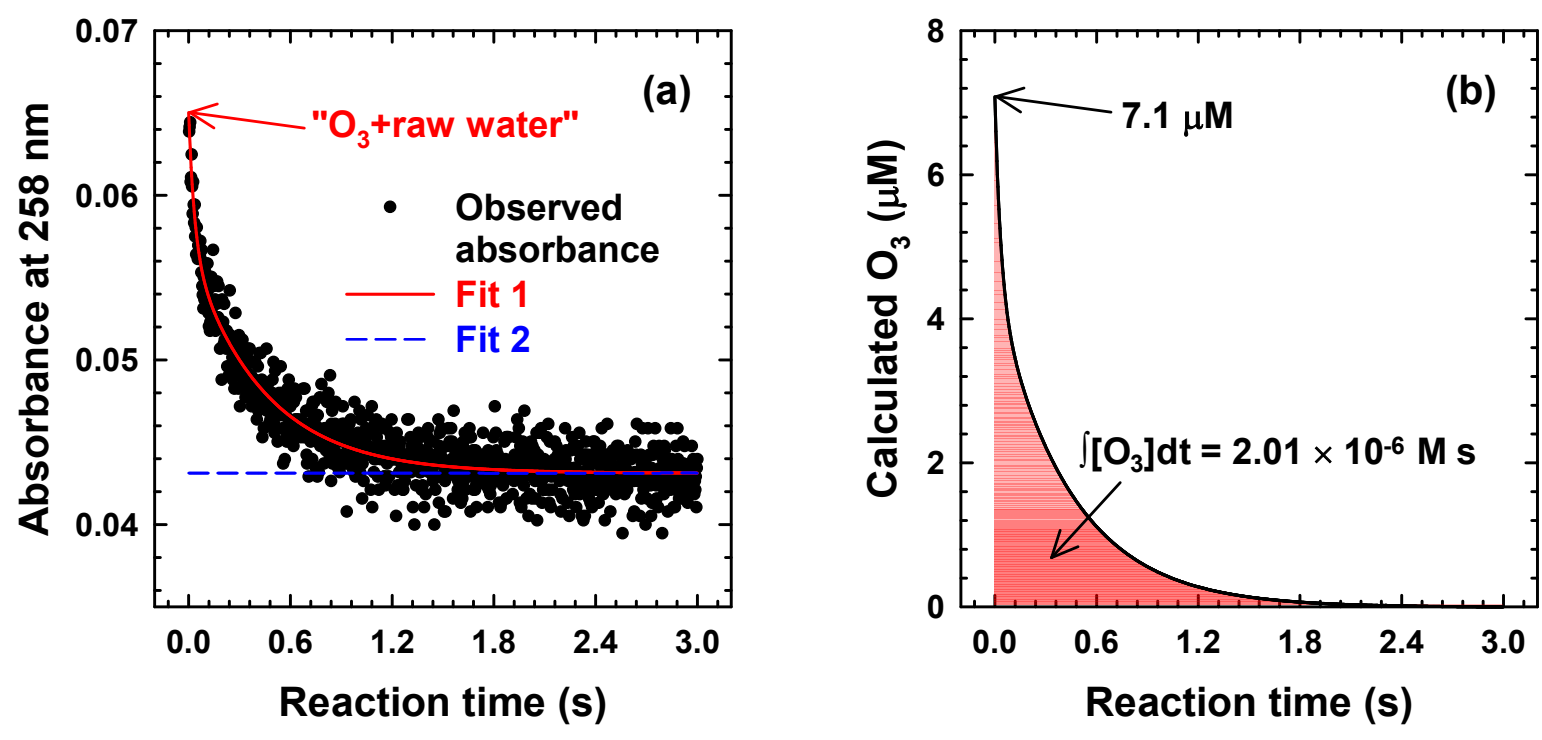

Figure S10. (a) Absorbance at $258 \mathrm{~nm}$ during $\mathrm{O}_{3}$ decomposition in the Gamak natural water. Red and bule dotted lines represent fits of the absorbance regarding whole examined reaction time and stationary phases (from 2 to 3 s), respectively. (b) Calculated $\mathrm{O}_{3}$ from the subtraction of blue dotted line from red line in (a) $\left(\left[\mathrm{O}_{3}\right]_{0}=7.3 \mu \mathrm{M}, \mathrm{pH}=7.95\right.$, temperature $=$ $\left.20 \pm 1^{\circ} \mathrm{C}\right)$. 

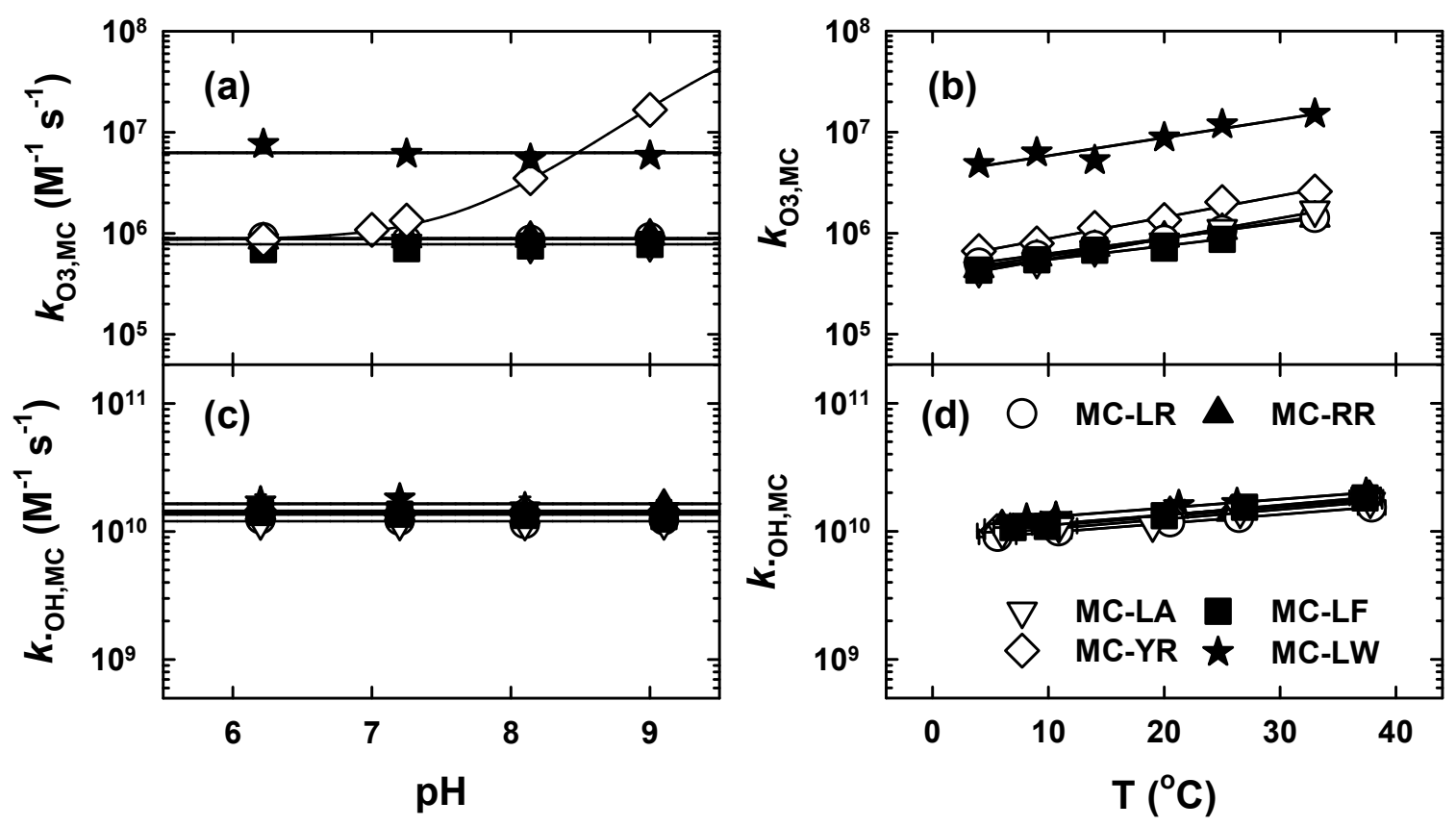

Figure S11. Effects of $\mathrm{pH}$ and temperature on $(\mathrm{a}, \mathrm{b}) k_{\mathrm{O}, \mathrm{MC}}$ and $(\mathrm{c}, \mathrm{d}) k_{\bullet \mathrm{OH}, \mathrm{MC}}\left(20 \pm 1{ }^{\circ} \mathrm{C}\right.$ for (a) and (c), $\mathrm{pH}=7.2$ for (b) and (d)). 

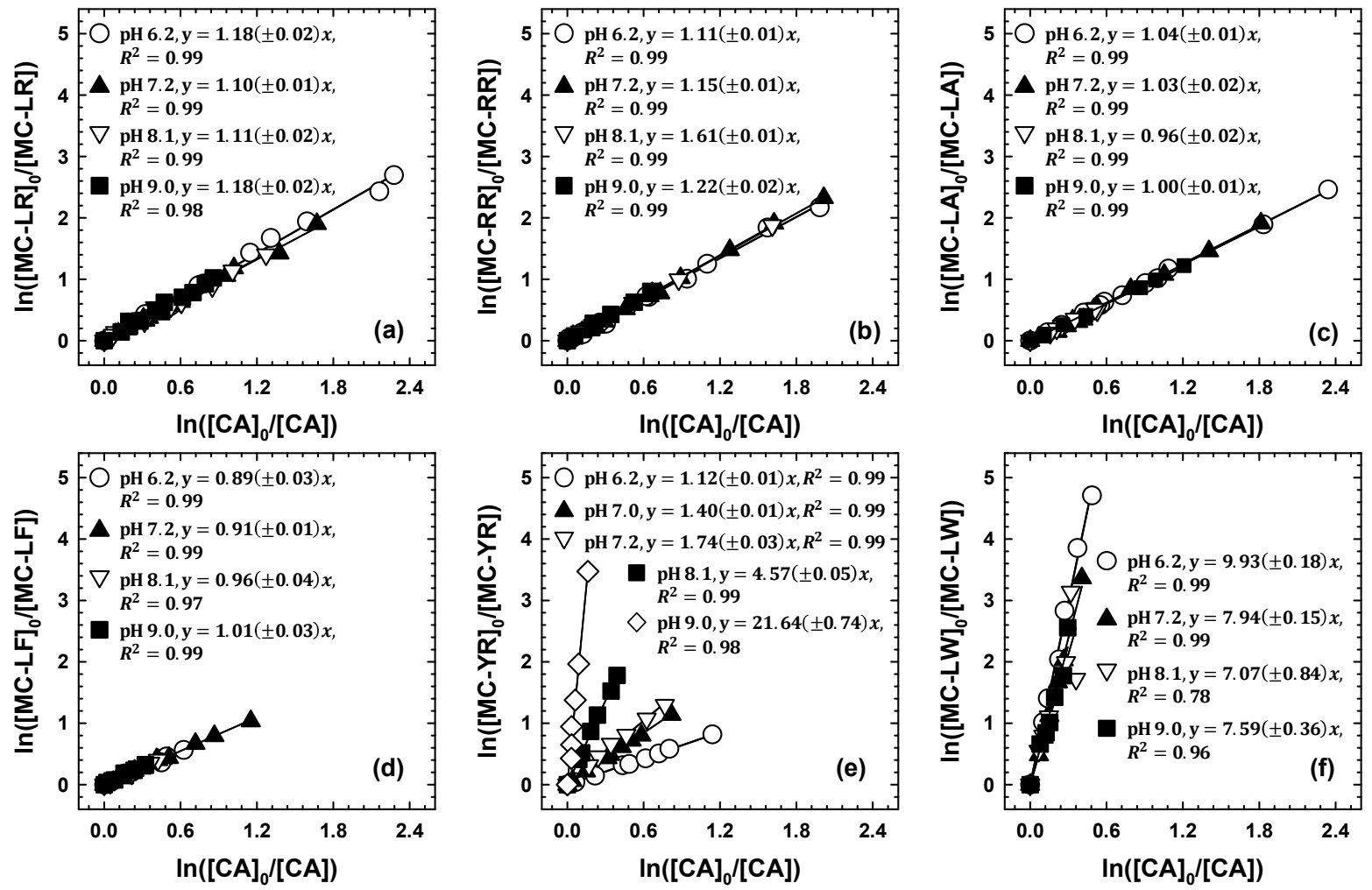

Figure S12. CK plots for the determination of $k_{\mathrm{O} 3, \mathrm{MC}}$ at different $\mathrm{pH}$ values $\left([\mathrm{MC}]_{0}=0.1 \mu \mathrm{M}\right.$, $[\mathrm{CA}]_{0}=0.1 \mu \mathrm{M},\left[\mathrm{O}_{3}\right]_{0}=0.025-0.25 \mu \mathrm{M},[t-\mathrm{BuOH}]_{0}=5 \mathrm{mM}$, temperature $\left.=20 \pm 1^{\circ} \mathrm{C}\right)$. 

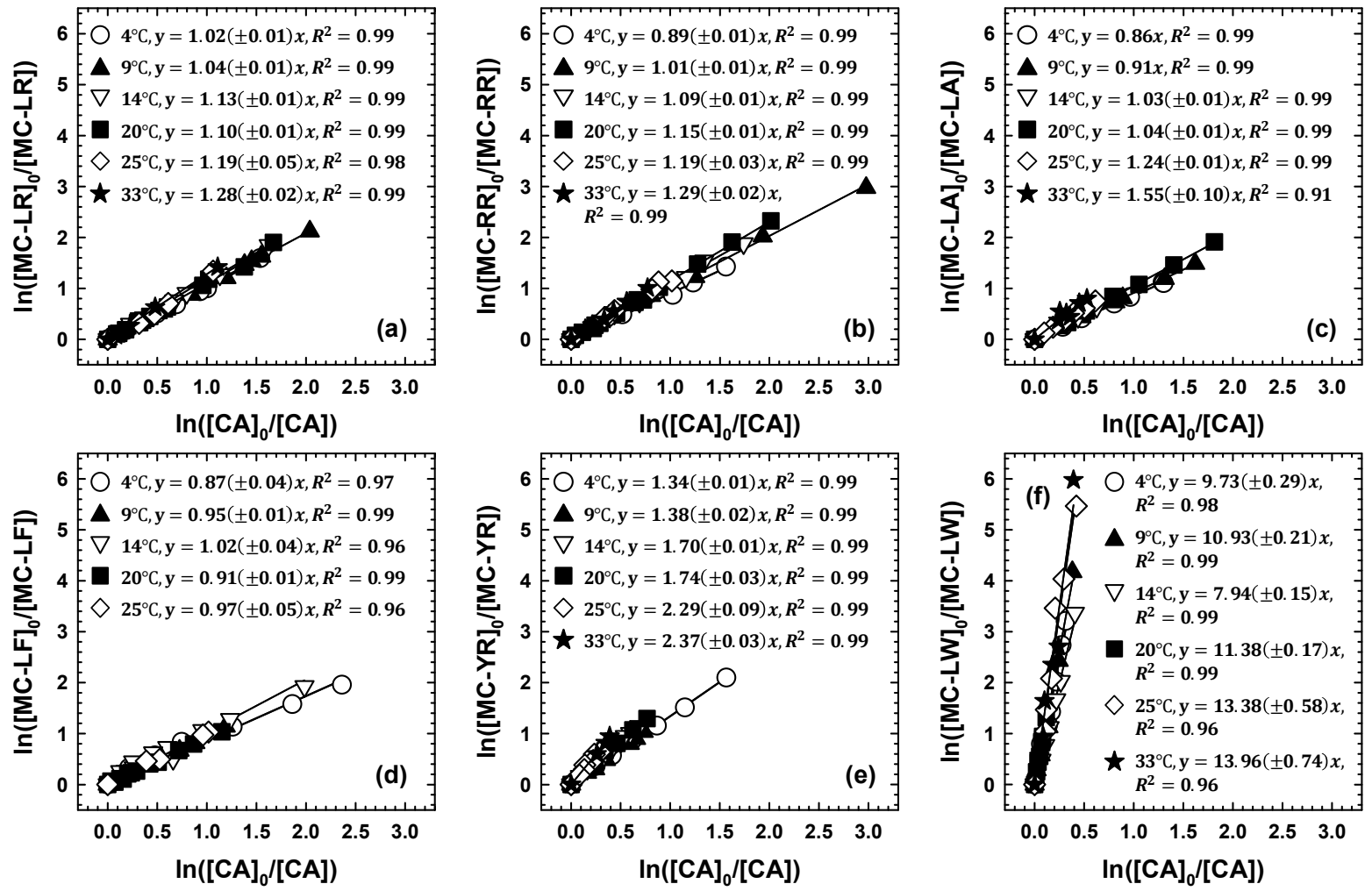

Figure S13. CK plots for the determination of $k_{\mathrm{O} 3, \mathrm{MC}}$ at different temperatures $\left([\mathrm{MC}]_{0}=0.1\right.$ $\left.\mu \mathrm{M},[\mathrm{CA}]_{0}=0.1 \mu \mathrm{M},\left[\mathrm{O}_{3}\right]_{0}=0.025-0.25 \mu \mathrm{M},[t-\mathrm{BuOH}]_{0}=5 \mathrm{mM}, \mathrm{pH}=7.2\right)$. 


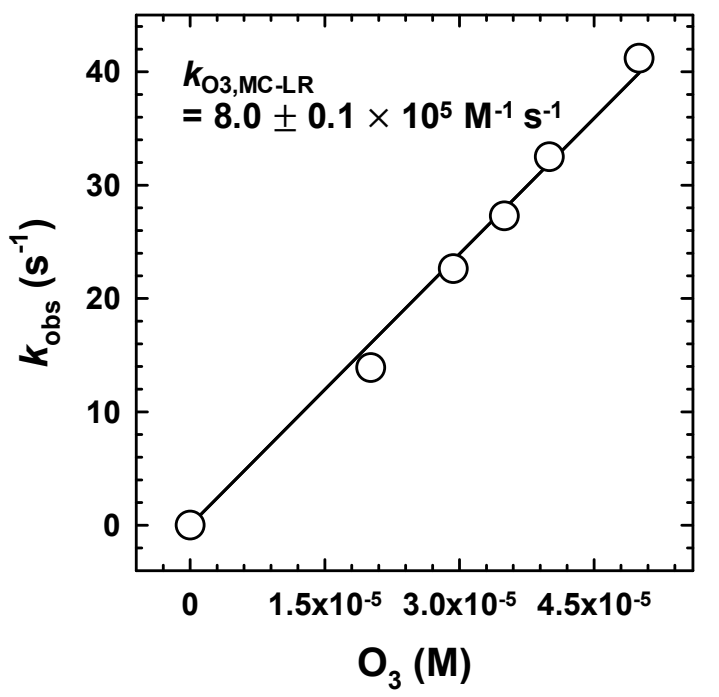

Figure S14. Plot of pseudo-first-order rate constants of MC-LR decomposition $\left(k_{\mathrm{obs}}\right)$ as a function of $\mathrm{O}_{3}$ concentration $\left([\mathrm{MC}-\mathrm{LR}]_{0}=5 \mu \mathrm{M},[t-\mathrm{BuOH}]_{0}=5 \mathrm{mM}\right.$, temperature $=20 \pm 1^{\circ} \mathrm{C}$, $\mathrm{pH}=7.2)$. 

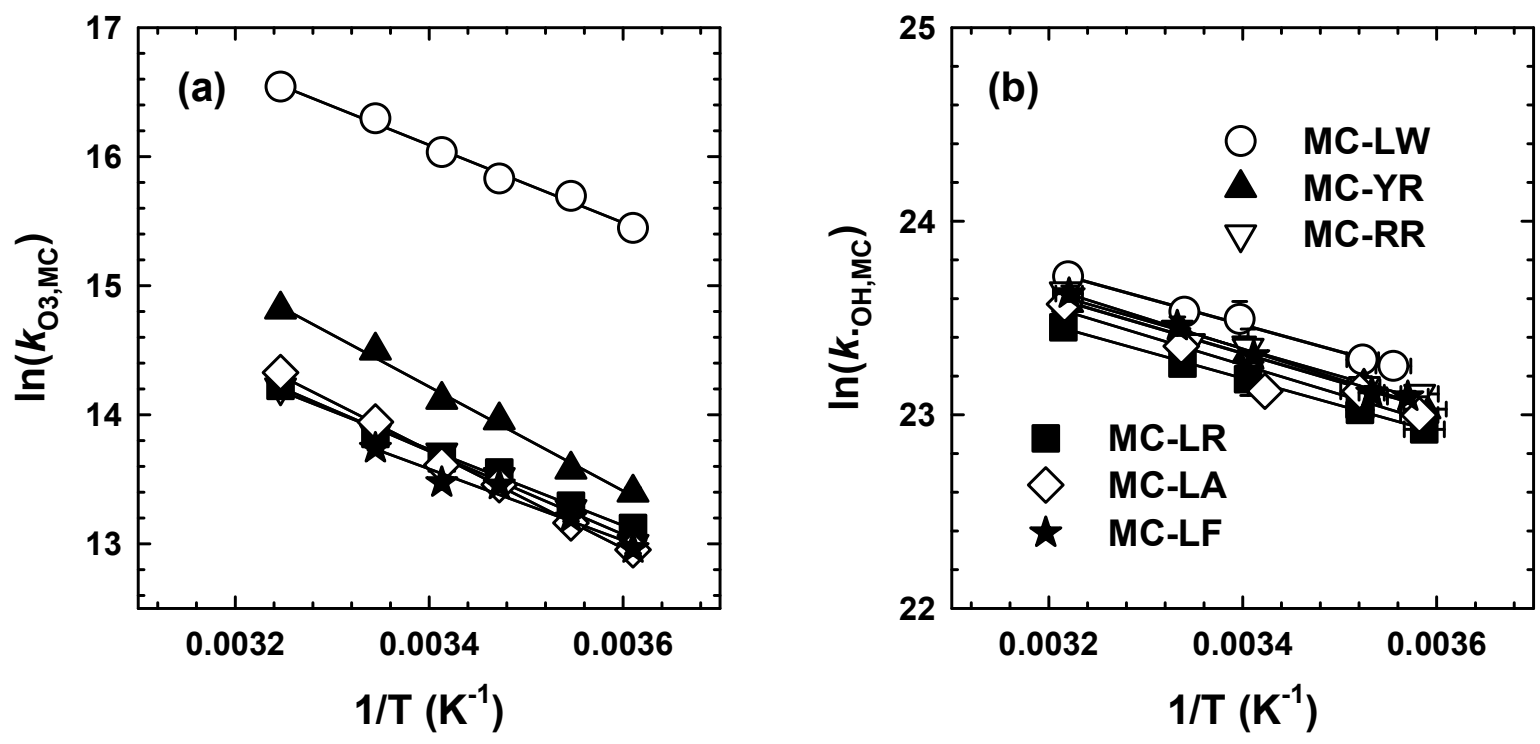

Figure S15. Arrhenius plots of (a) $k_{\mathrm{O} 3, \mathrm{MC}}$ and (b) $k_{\bullet} \mathrm{OH}, \mathrm{MC}(\mathrm{pH}=7.2)$. 

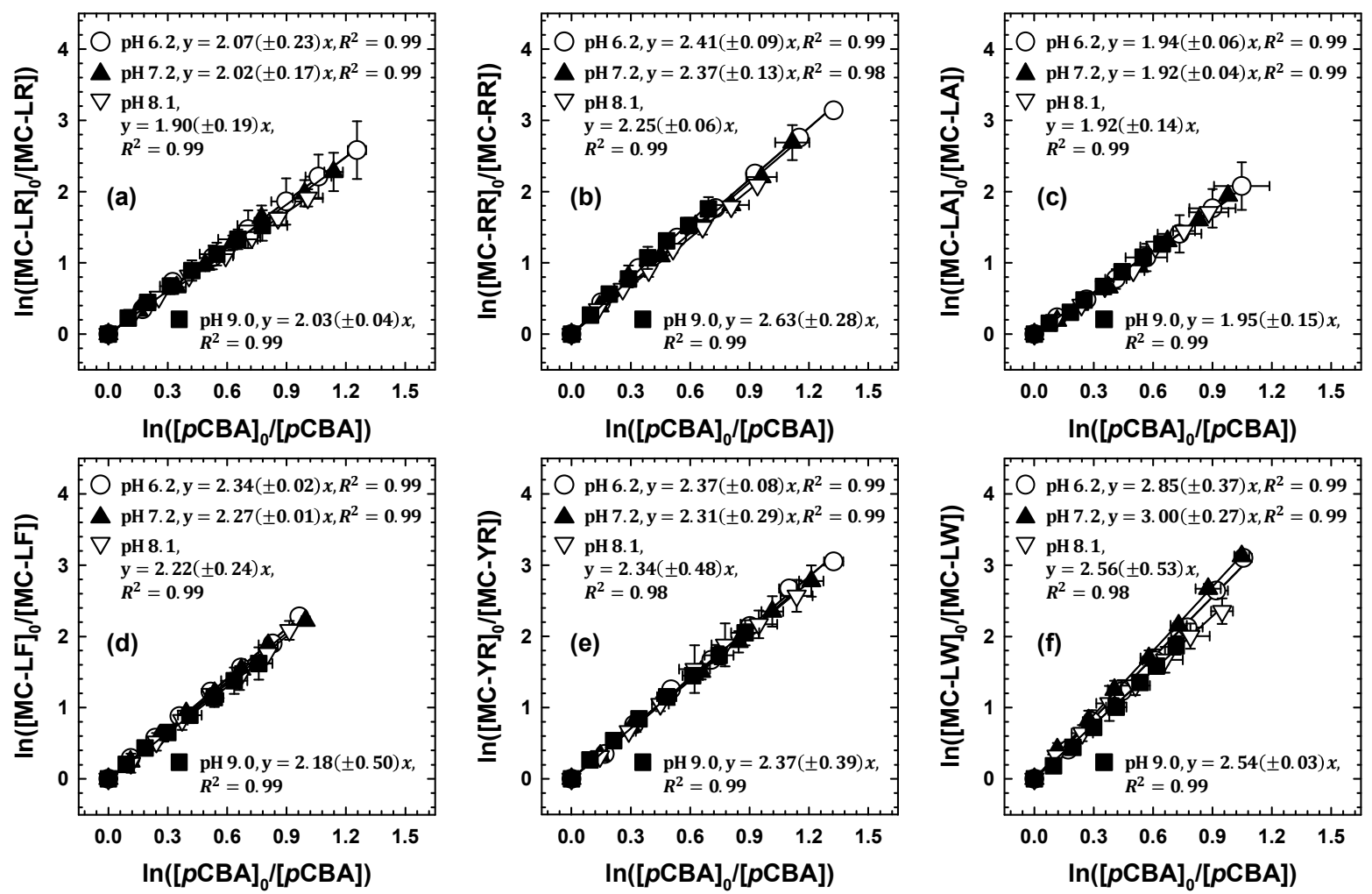

Figure S16. CK plots for the determination of $k_{\bullet} \mathrm{OH}, \mathrm{MC}$ at different $\mathrm{pH}$ values $\left([\mathrm{MC}]_{0}=0.1\right.$ $\mu \mathrm{M},[p \mathrm{CBA}]_{0}=0.1 \mu \mathrm{M},\left[\mathrm{H}_{2} \mathrm{O}_{2}\right]_{0}=1 \mathrm{mM}$, temperature $\left.=20 \pm 1^{\circ} \mathrm{C}\right)$. 

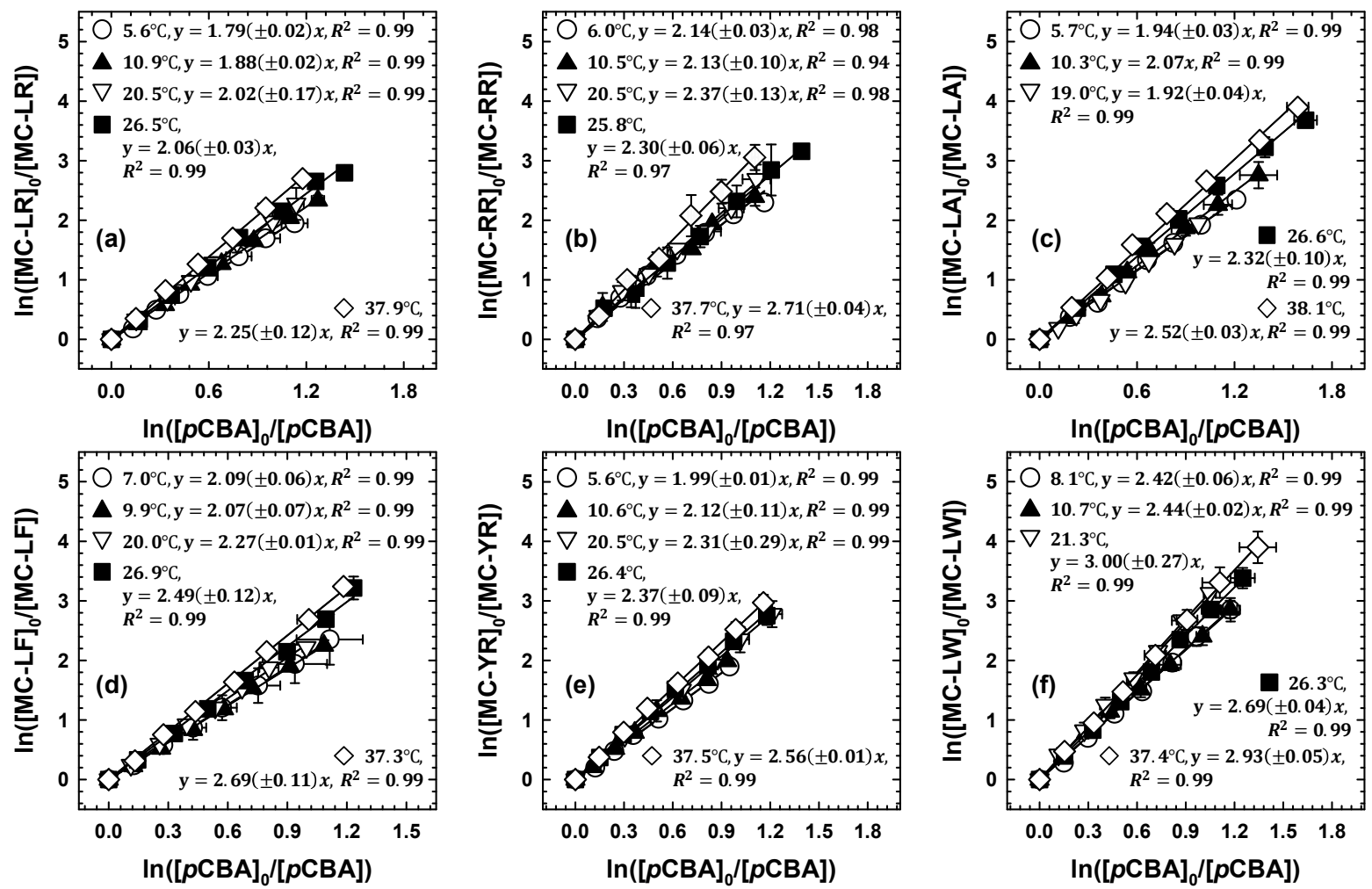

Figure S17. CK plots for the determination of $k_{\bullet} \mathrm{OH}, \mathrm{MC}$ at different temperatures $\left([\mathrm{MC}]_{0}=0.1\right.$ $\left.\mu \mathrm{M},[p \mathrm{CBA}]_{0}=0.1 \mu \mathrm{M},\left[\mathrm{H}_{2} \mathrm{O}_{2}\right]_{0}=1 \mathrm{mM}, \mathrm{pH}=7.2\right)$. 

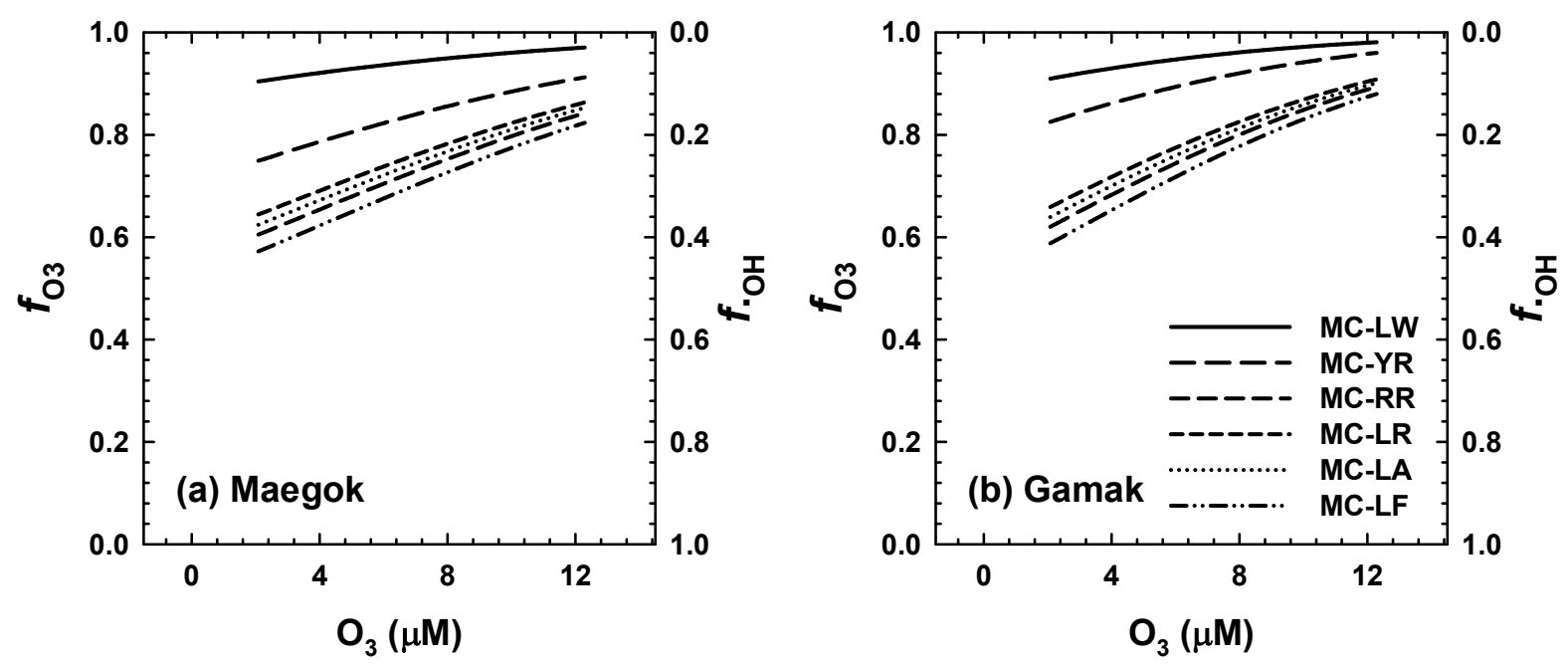

Figure S18. Contribution of $\mathrm{O}_{3}\left(f_{\mathrm{O} 3}\right)$ and ${ }^{\bullet} \mathrm{OH}\left(f_{\bullet} \cdot \mathrm{OH}\right)$ to the overall oxidation of MCs in (a) Maegok and (b) Gamak natural waters as a function of the initial $\mathrm{O}_{3}$ does. $f_{\mathrm{O} 3}$ and $f_{\bullet \mathrm{OH}}$ are defined as $k_{\mathrm{O} 3, \mathrm{MC}} \int\left[\mathrm{O}_{3}\right] \mathrm{dt} /\left(k_{\mathrm{O} 3, \mathrm{MC}} \int\left[\mathrm{O}_{3}\right] \mathrm{dt}+k_{\bullet \mathrm{OH}, \mathrm{MC}} \int\left[{ }^{\circ} \mathrm{OH}\right] \mathrm{dt}\right)$ and $1-f_{\mathrm{O} 3}$, respectively $\left([\mathrm{MC}]_{0}=\right.$ $0.1 \mu \mathrm{M},[p \mathrm{CBA}]_{0}=0.1 \mu \mathrm{M}$, temperature $\left.=20 \pm 1^{\circ} \mathrm{C}\right)$. 
MC-LR, [M+H] ${ }^{+}$formula: $\mathrm{C}_{49} \mathrm{H}_{75} \mathrm{~N}_{10} \mathrm{O}_{12}$, Theoretical m/z: 995.5560
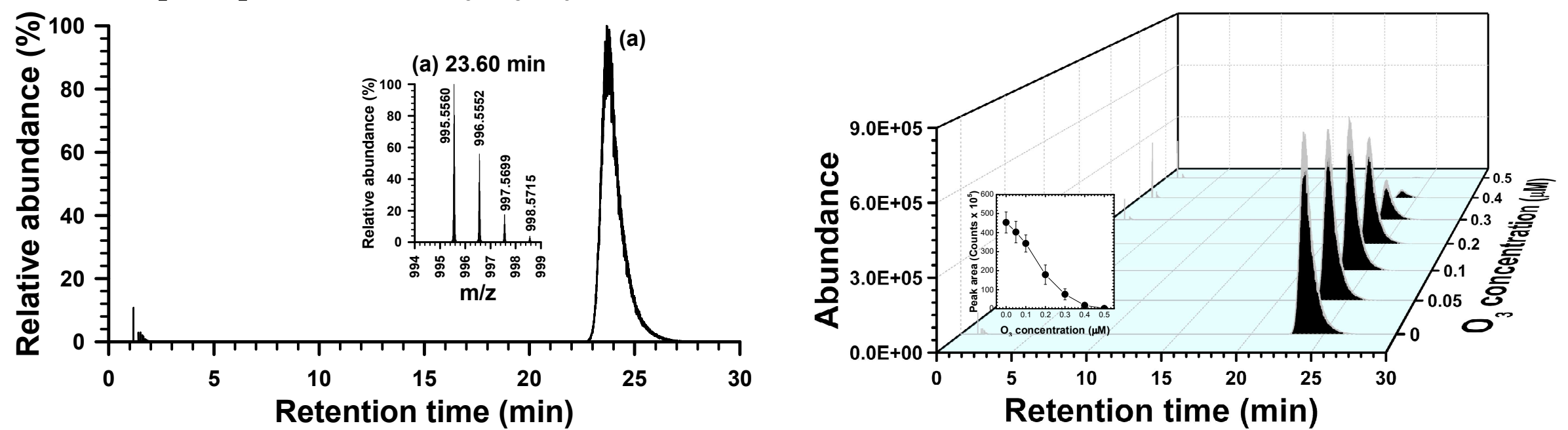

Figure S19. LC/MS identification of MC-LR $\left([\mathrm{M}+\mathrm{H}]^{+}=995.5560\right)$. The chromatogram at $0 \mu \mathrm{M}$ of $\mathrm{O}_{3}$ with mass spectra (left) and $\mathrm{O}_{3}$ concentration-dependent chromatograms (right) of MC-LR. 
PLR1, [M+H] ${ }^{+}$formula: $\mathrm{C}_{34} \mathrm{H}_{55} \mathrm{~N}_{10} \mathrm{O}_{12}$, Theoretical m/z: 795.3995
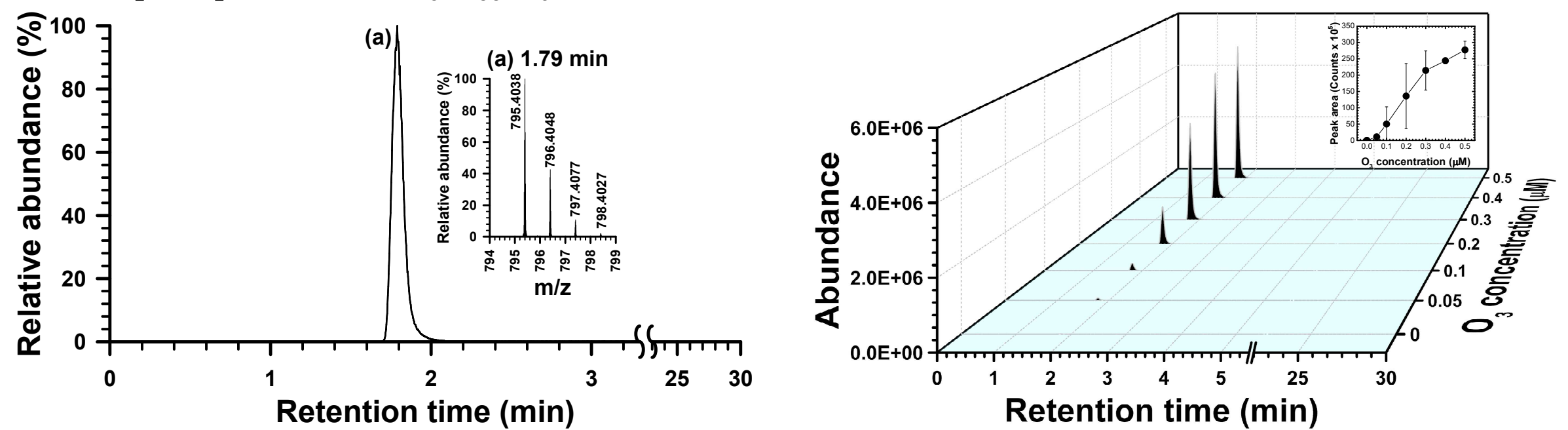

Figure S20. LC/MS identification of PLR1 $\left([\mathrm{M}+\mathrm{H}]^{+}=795.3995\right)$. The chromatogram at $0.5 \mu \mathrm{M}$ of $\mathrm{O}_{3}$ with mass spectra (left) and $\mathrm{O}_{3}$ concentration-dependent chromatograms (right) of PLR1. 
PLR2, [M+H] ${ }^{+}$formula: $\mathrm{C}_{37} \mathrm{H}_{59} \mathrm{~N}_{10} \mathrm{O}_{12}$, Theoretical m/z: 835.4308
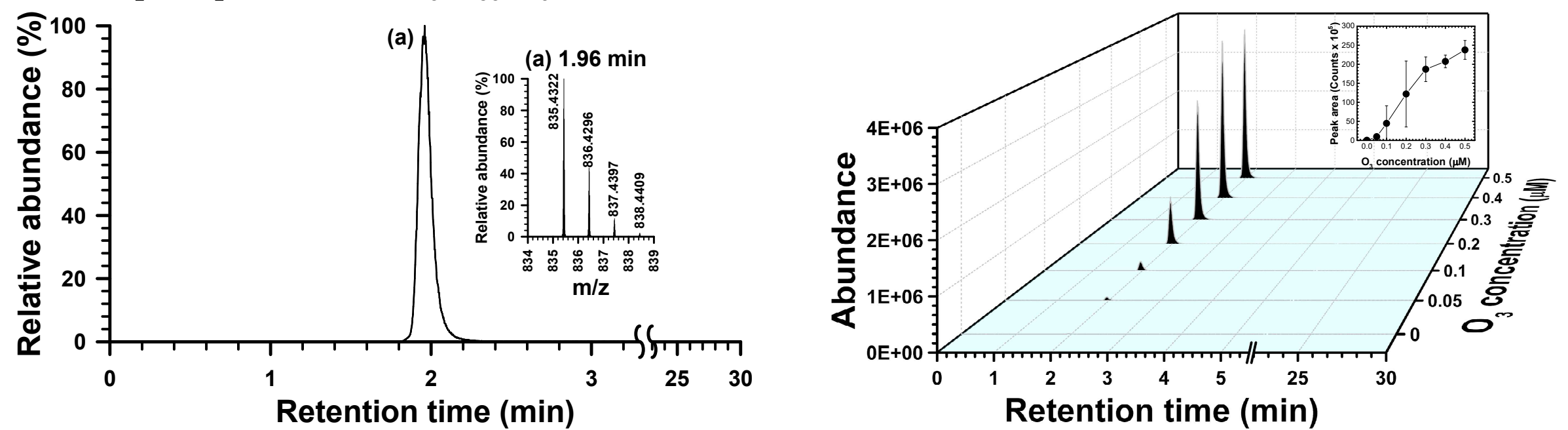

Figure S21. LC/MS identification of PLR2 $\left([\mathrm{M}+\mathrm{H}]^{+}=835.4308\right)$. The chromatogram at $0.5 \mu \mathrm{M}$ of $\mathrm{O}_{3}$ with mass spectra (left) and $\mathrm{O}_{3}$ concentration-dependent chromatograms (right) of PLR2. 
MC-RR, [M+H] ${ }^{+}$formula: $\mathrm{C}_{49} \mathrm{H}_{76} \mathrm{~N}_{13} \mathrm{O}_{12}$, Theoretical m/z: 1038.5730
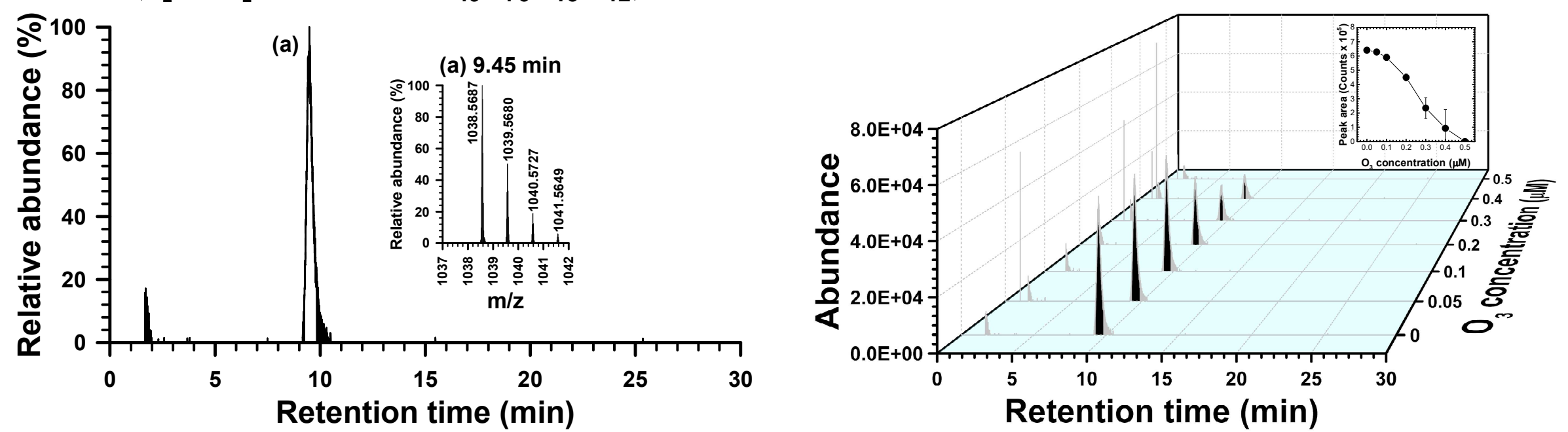

Figure S22. $\mathrm{LC} / \mathrm{MS}$ identification of $\mathrm{MC}-\mathrm{RR}\left([\mathrm{M}+\mathrm{H}]^{+}=1038.5730\right)$. The chromatogram at $0 \mu \mathrm{M}$ of $\mathrm{O}_{3}$ with mass spectra (left) and $\mathrm{O}_{3}$ concentration-dependent chromatograms (right) of MC-RR. 
PRR1, [M+H] $]^{+}$formula: $\mathrm{C}_{34} \mathrm{H}_{56} \mathrm{~N}_{13} \mathrm{O}_{12}$, Theoretical m/z: 838.4165
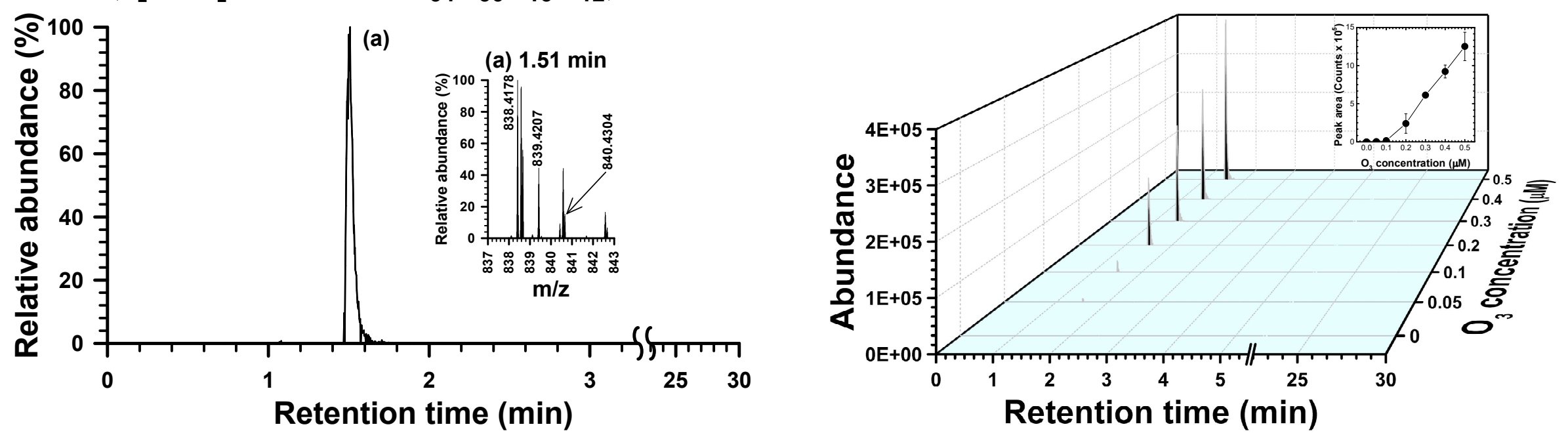

Figure S23. LC/MS identification of PRR1 $\left([\mathrm{M}+\mathrm{H}]^{+}=838.4165\right)$. The chromatogram at $0.5 \mu \mathrm{M}$ of $\mathrm{O}_{3}$ with mass spectra (left) and $\mathrm{O}_{3}$ concentration-dependent chromatograms (right) of PRR1. 
PRR2, [M+H] $]^{+}$formula: $\mathrm{C}_{37} \mathrm{H}_{60} \mathrm{~N}_{13} \mathrm{O}_{12}$, Theoretical m/z: 878.4478
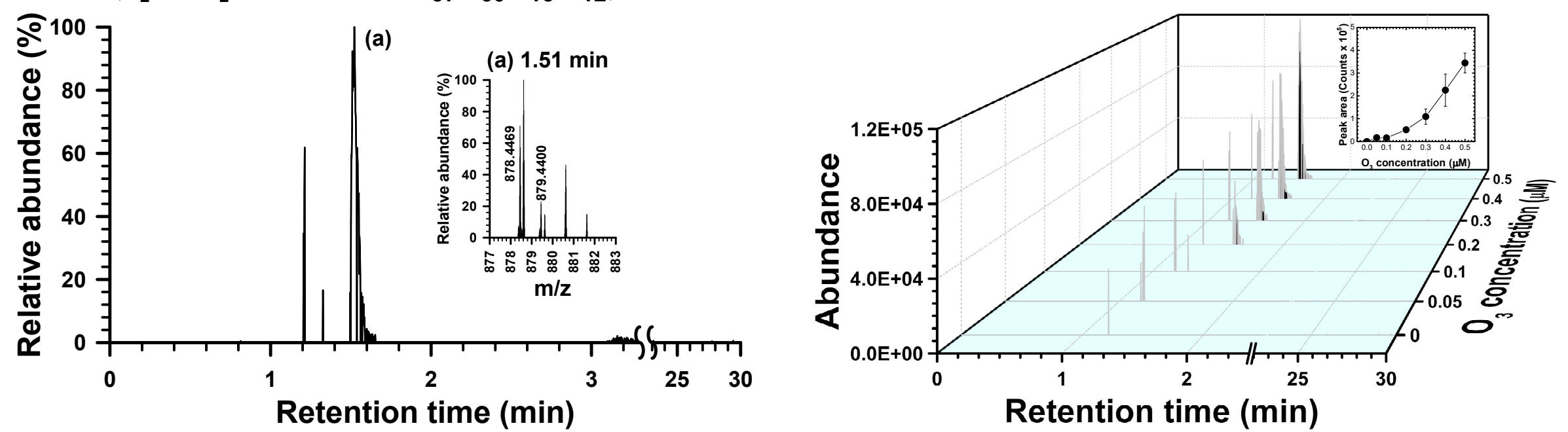

Figure S24. LC/MS identification of PRR2 $\left([\mathrm{M}+\mathrm{H}]^{+}=878.4478\right)$. The chromatogram at $0.5 \mu \mathrm{M}$ of $\mathrm{O}_{3}$ with mass spectra (left) and $\mathrm{O}_{3}$ concentration-dependent chromatograms (right) of PRR2. 
MC-LA, [M+H] ${ }^{+}$formula: $\mathrm{C}_{46} \mathrm{H}_{68} \mathrm{~N}_{7} \mathrm{O}_{12}$, Theoretical m/z: 910.4920
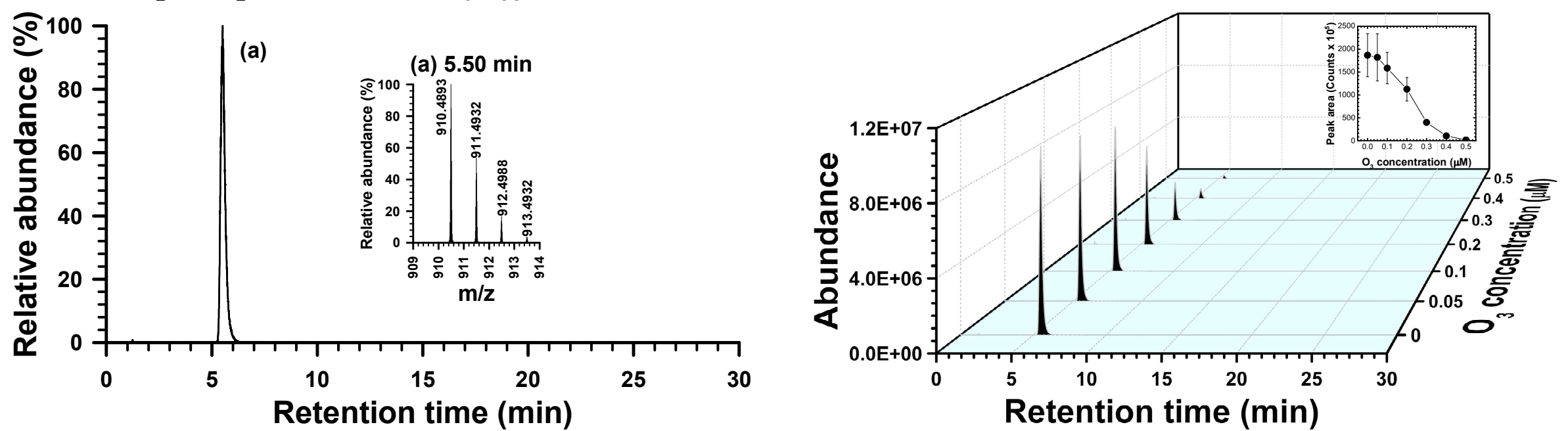

Figure S25. LC/MS identification of MC-LA $\left([\mathrm{M}+\mathrm{H}]^{+}=910.4920\right)$. The chromatogram at $0 \mu \mathrm{M}$ of $\mathrm{O}_{3}$ with mass spectra (left) and $\mathrm{O}_{3}$ concentration-dependent chromatograms (right) of MC-LA. 
PLA1, [M+H] ${ }^{+}$formula: $\mathrm{C}_{31} \mathrm{H}_{48} \mathrm{~N}_{7} \mathrm{O}_{12}$, Theoretical m/z: 710.3355
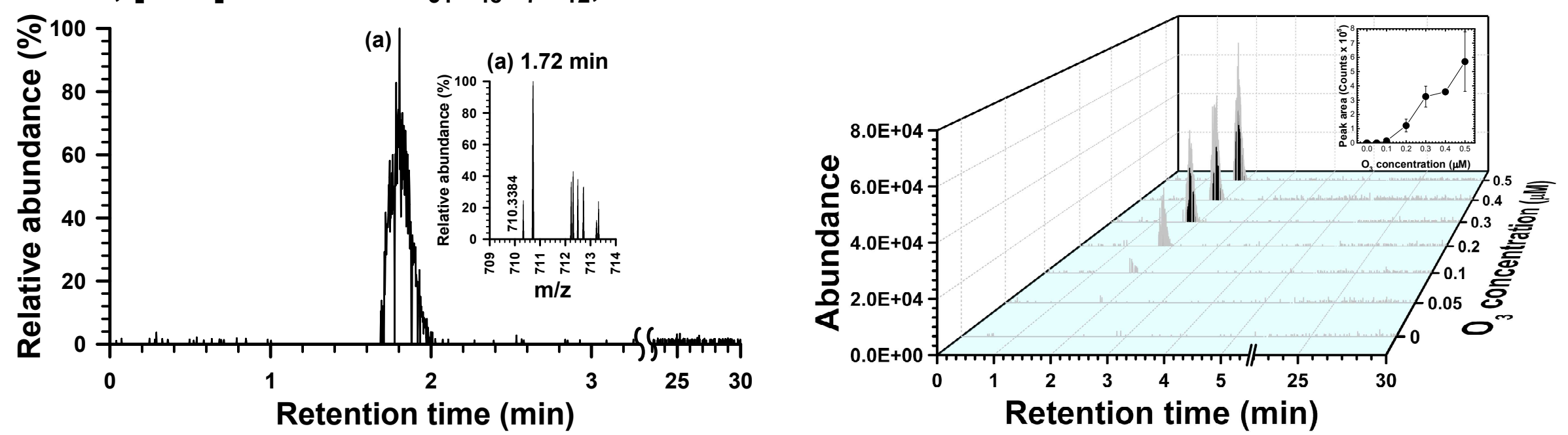

Figure S26. LC/MS identification of PLA1 $\left([\mathrm{M}+\mathrm{H}]^{+}=710.3355\right)$. The chromatogram at $0.5 \mu \mathrm{M}$ of $\mathrm{O}_{3}$ with mass spectra (left) and $\mathrm{O}_{3}$ concentration-dependent chromatograms (right) of PLA1. 
PLA2, $[M+H]^{+}$formula: $\mathrm{C}_{34} \mathrm{H}_{52} \mathrm{~N}_{7} \mathrm{O}_{12}$, Theoretical m/z: 750.3668
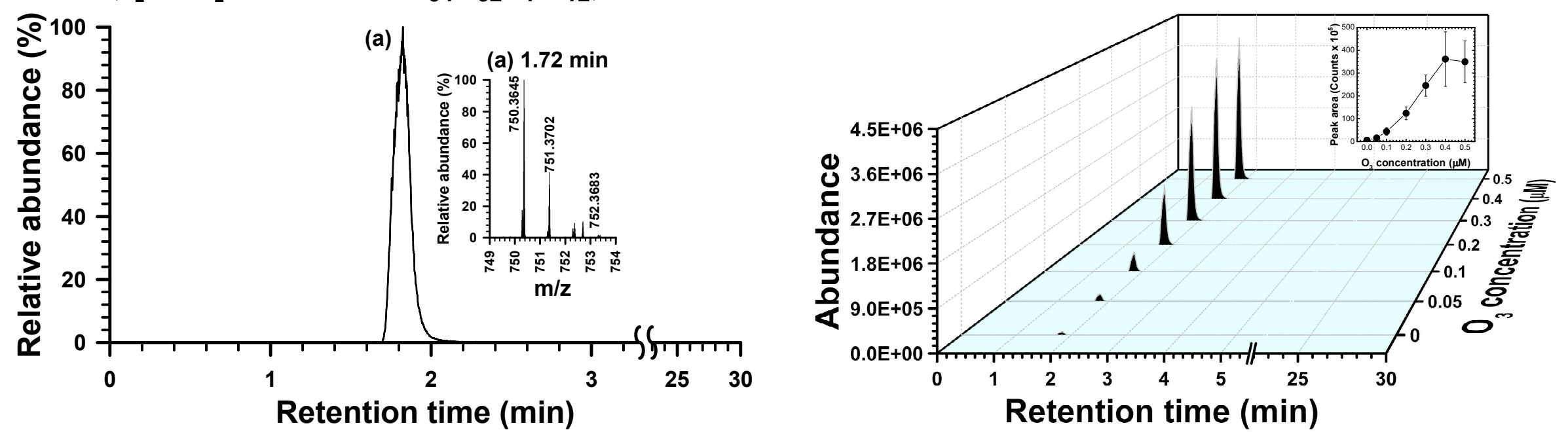

Figure S27. LC/MS identification of PLA2 $\left([\mathrm{M}+\mathrm{H}]^{+}=750.3668\right)$. The chromatogram at $0.5 \mu \mathrm{M}$ of $\mathrm{O}_{3}$ with mass spectra (left) and $\mathrm{O}_{3}$ concentration-dependent chromatograms (right) of P750. 
MC-LF, $[M+H]^{+}$formula: $\mathrm{C}_{52} \mathrm{H}_{72} \mathrm{~N}_{7} \mathrm{O}_{12}$, Theoretical m/z: 986.5233
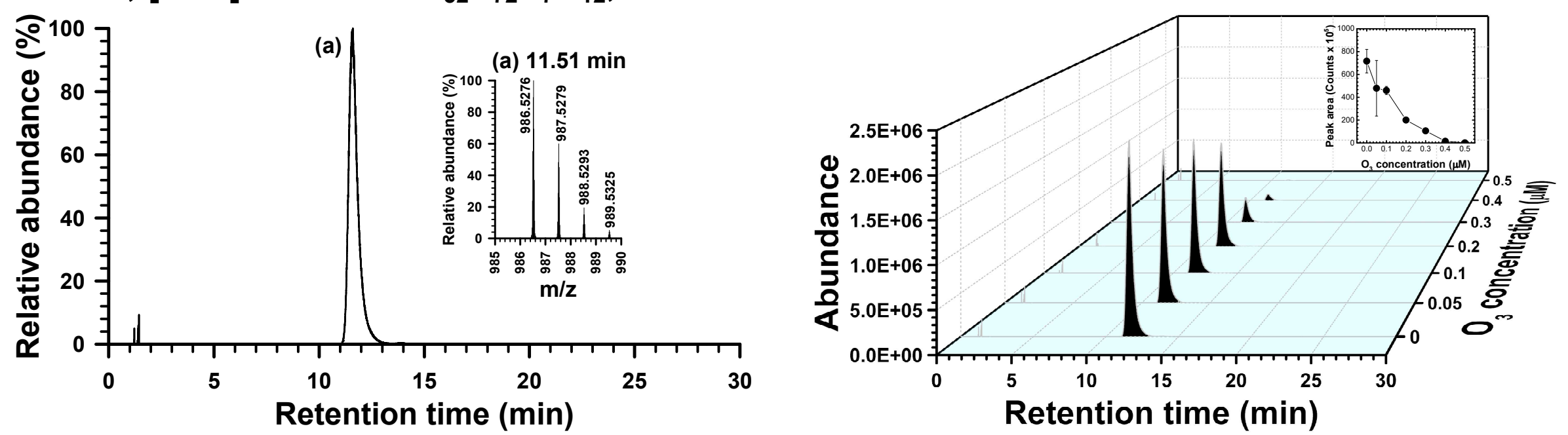

Figure S28. LC/MS identification of MC-LF $\left([\mathrm{M}+\mathrm{H}]^{+}=986.5233\right)$. The chromatogram at $0 \mu \mathrm{M}$ of $\mathrm{O}_{3}$ with mass spectra (left) and $\mathrm{O}_{3}$ concentration-dependent chromatograms (right) of MC-LF. 
PLF1, [M+H] ${ }^{+}$formula: $\mathrm{C}_{37} \mathrm{H}_{52} \mathrm{~N}_{7} \mathrm{O}_{12}$, Theoretical m/z: 786.3668
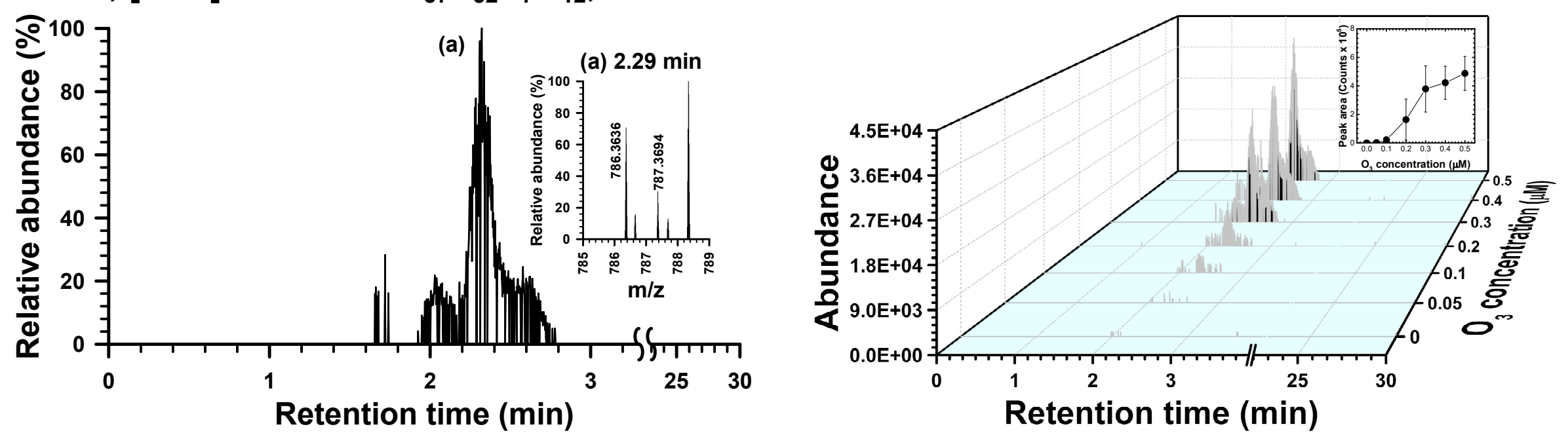

Figure S29. LC/MS identification of PLF1 $\left([\mathrm{M}+\mathrm{H}]^{+}=786.3668\right)$. The chromatogram at $0.5 \mu \mathrm{M}$ of $\mathrm{O}_{3}$ with mass spectra (left) and $\mathrm{O}_{3}$ concentration-dependent chromatograms (right) of PLF1. 
PLF2, $[\mathrm{M}+\mathrm{H}]^{+}$formula: $\mathrm{C}_{40} \mathrm{H}_{56} \mathrm{~N}_{7} \mathrm{O}_{12}$, Theoretical m/z: 826.3981
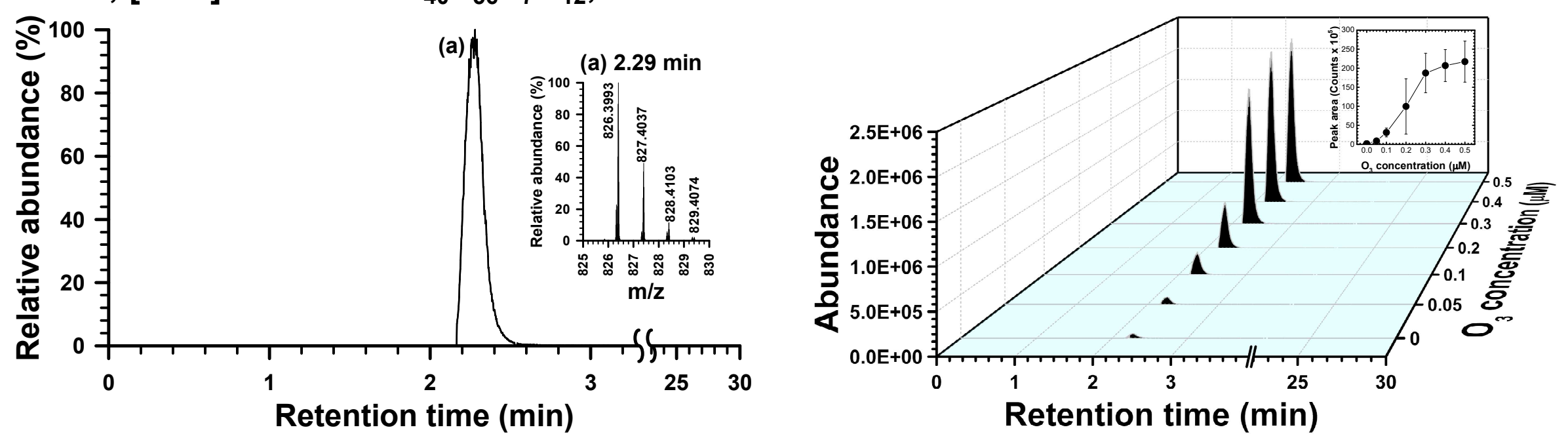

Figure S30. LC/MS identification of PLF2 $\left([\mathrm{M}+\mathrm{H}]^{+}=786.3668\right)$. The chromatogram at $0.5 \mu \mathrm{M}$ of $\mathrm{O}_{3}$ with mass spectra (left) and $\mathrm{O}_{3}$ concentration-dependent chromatograms (right) of PLF2. 
MC-YR, [M+H] ${ }^{+}$formula: $\mathrm{C}_{52} \mathrm{H}_{73} \mathrm{~N}_{10} \mathrm{O}_{13}$, Theoretical m/z: 1045.5353
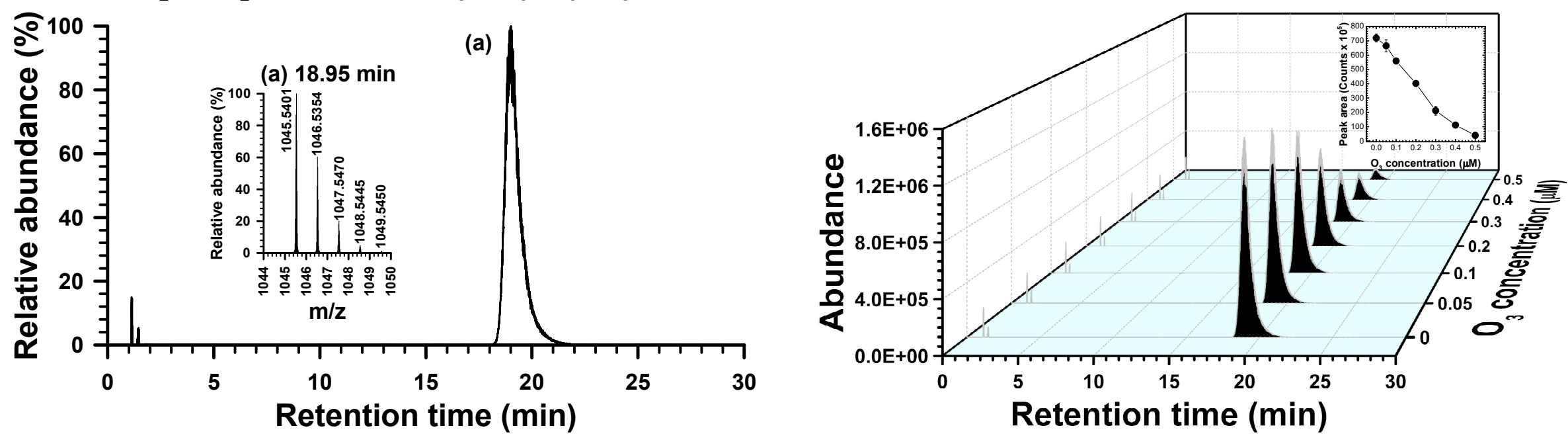

Figure S31. LC/MS identification of MC-YR $\left([\mathrm{M}+\mathrm{H}]^{+}=1045.5353\right)$. The chromatogram at $0 \mu \mathrm{M}$ of $\mathrm{O}_{3}$ with mass spectra (left) and $\mathrm{O}_{3}$ concentration-dependent chromatograms (right) of MC-YR. 
PYR1, [M+H] ${ }^{+}$formula: $\mathrm{C}_{37} \mathrm{H}_{51} \mathrm{~N}_{10} \mathrm{O}_{14}$, Theoretical m/z: 859.3580
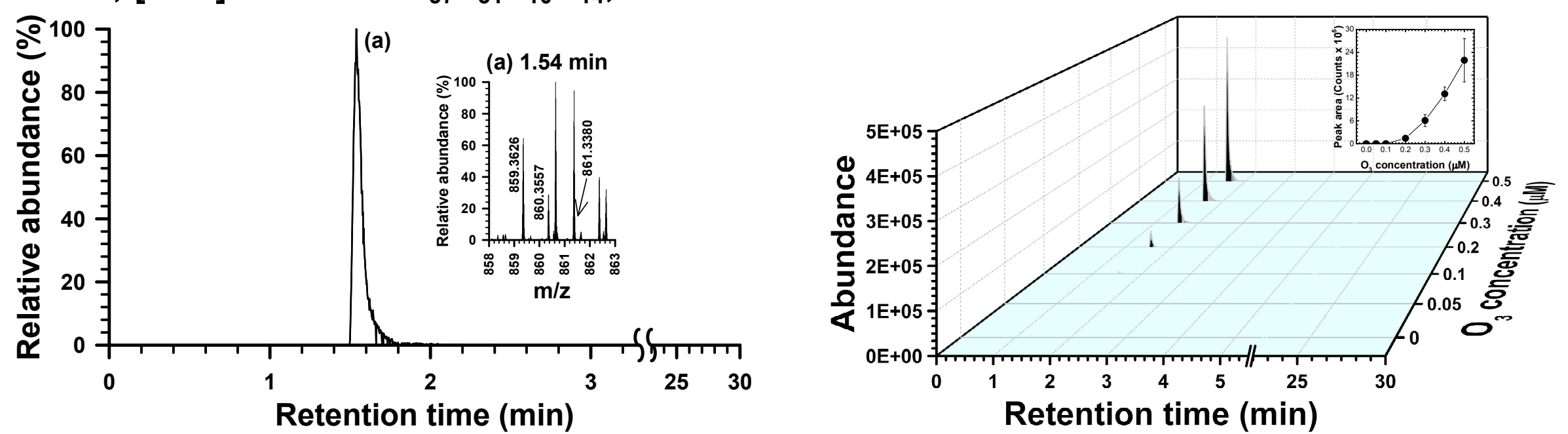

Figure S32. LC/MS identification of PYR1 $\left([\mathrm{M}+\mathrm{H}]^{+}=859.3580\right)$. The chromatogram at $0.5 \mu \mathrm{M}$ of $\mathrm{O}_{3}$ with mass spectra (left) and $\mathrm{O}_{3}$ concentration-dependent chromatograms (right) of PYR1. 
PYR2, [M+H] ${ }^{+}$formula: $\mathrm{C}_{37} \mathrm{H}_{53} \mathrm{~N}_{10} \mathrm{O}_{14}$, Theoretical m/z: 861.3737
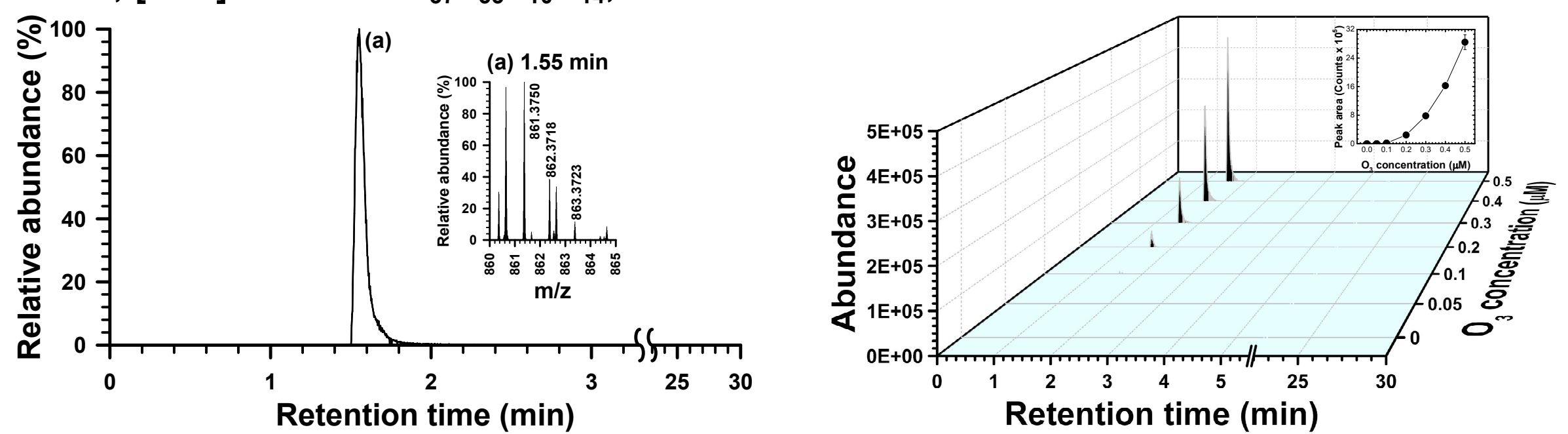

Figure S33. LC/MS identification of PYR2 $\left([\mathrm{M}+\mathrm{H}]^{+}=861.3737\right)$. The chromatogram at $0.5 \mu \mathrm{M}$ of $\mathrm{O}_{3}$ with mass spectra (left) and $\mathrm{O}_{3}$ concentration-dependent chromatograms (right) of PYR2. 
PYR3, [M+H] ${ }^{+}$formula: $\mathrm{C}_{40} \mathrm{H}_{55} \mathrm{~N}_{10} \mathrm{O}_{14}$, Theoretical m/z: 899.3893
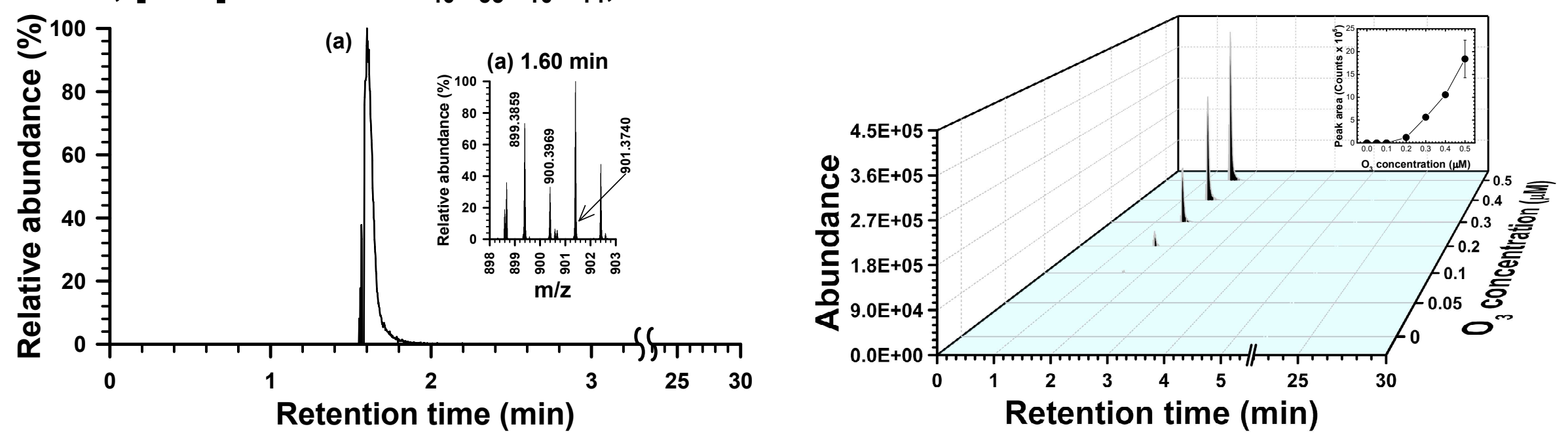

Figure S34. LC/MS identification of PYR3 $\left([\mathrm{M}+\mathrm{H}]^{+}=899.3893\right)$. The chromatogram at $0.5 \mu \mathrm{M}$ of $\mathrm{O}_{3}$ with mass spectra (left) and $\mathrm{O}_{3}$ concentration-dependent chromatograms (right) of PYR3. 
PYR4, [M+H] ${ }^{+}$formula: $\mathrm{C}_{40} \mathrm{H}_{57} \mathrm{~N}_{10} \mathrm{O}_{14}$, Theoretical m/z: 901.4050
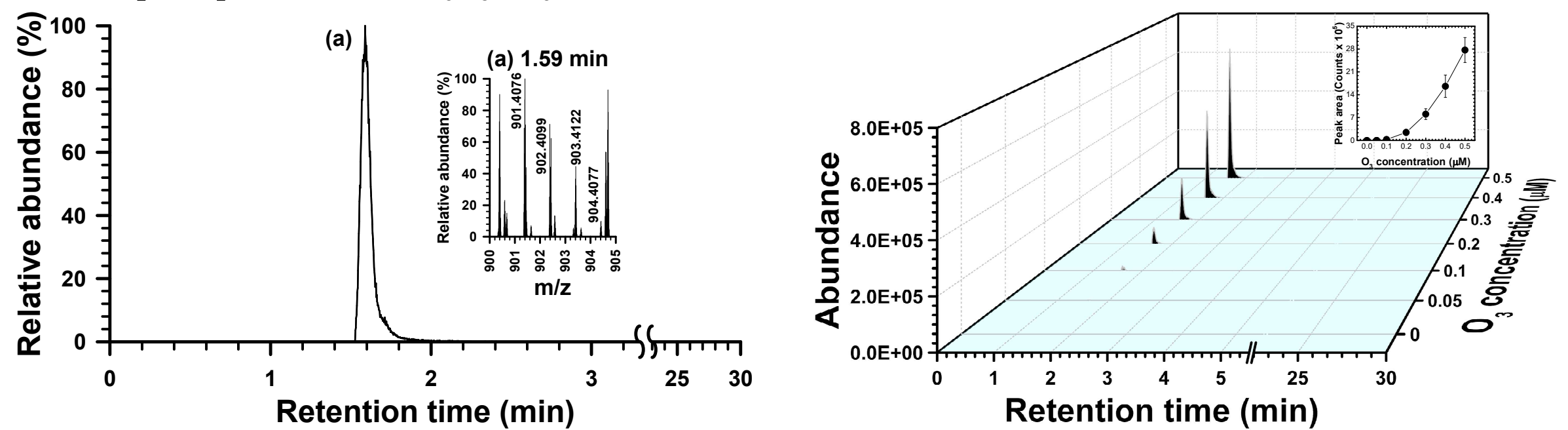

Figure S35. LC/MS identification of PYR4 $\left([\mathrm{M}+\mathrm{H}]^{+}=901.4050\right)$. The chromatogram at $0.5 \mu \mathrm{M}$ of $\mathrm{O}_{3}$ with mass spectra $($ left $)$ and $\mathrm{O}_{3}$ concentration-dependent chromatograms (right) of PYR4. 
PYR5, [M+H] ${ }^{+}$formula: $\mathrm{C}_{52} \mathrm{H}_{71} \mathrm{~N}_{10} \mathrm{O}_{14}$, Theoretical m/z: 1059.5145
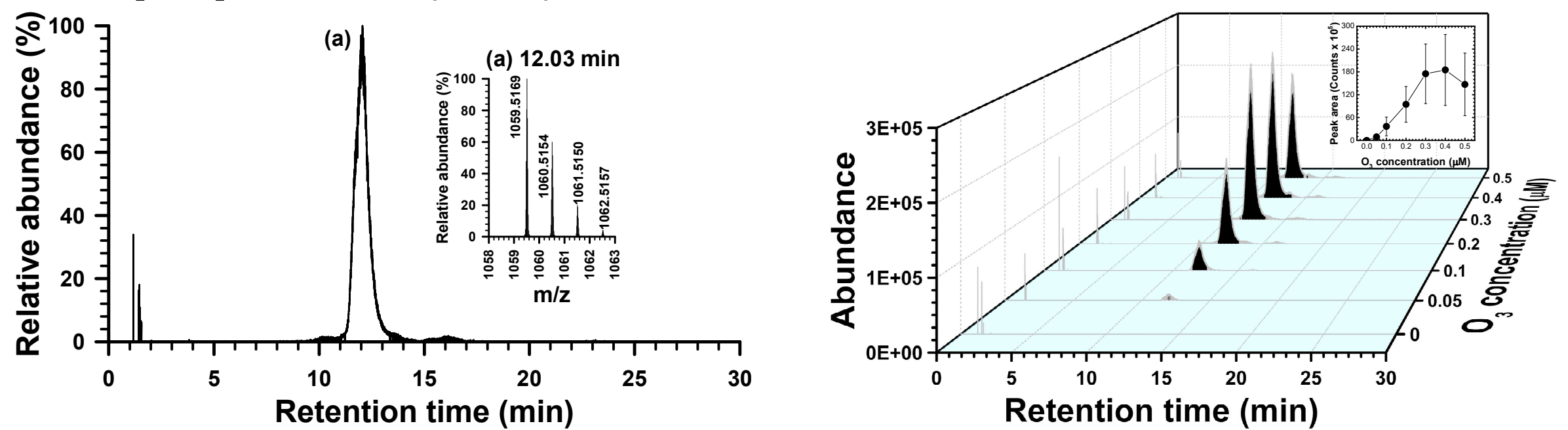

Figure S36. LC/MS identification of PYR5 $\left([\mathrm{M}+\mathrm{H}]^{+}=1059.5145\right)$. The chromatogram at $0.4 \mu \mathrm{M}$ of $\mathrm{O}_{3}$ with mass spectra $($ left $)$ and $\mathrm{O}_{3}$ concentration-dependent chromatograms (right) of PYR5. 
PYR6, [M+H] ${ }^{+}$formula: $\mathrm{C}_{52} \mathrm{H}_{73} \mathrm{~N}_{10} \mathrm{O}_{14}$, Theoretical m/z: 1061.5302
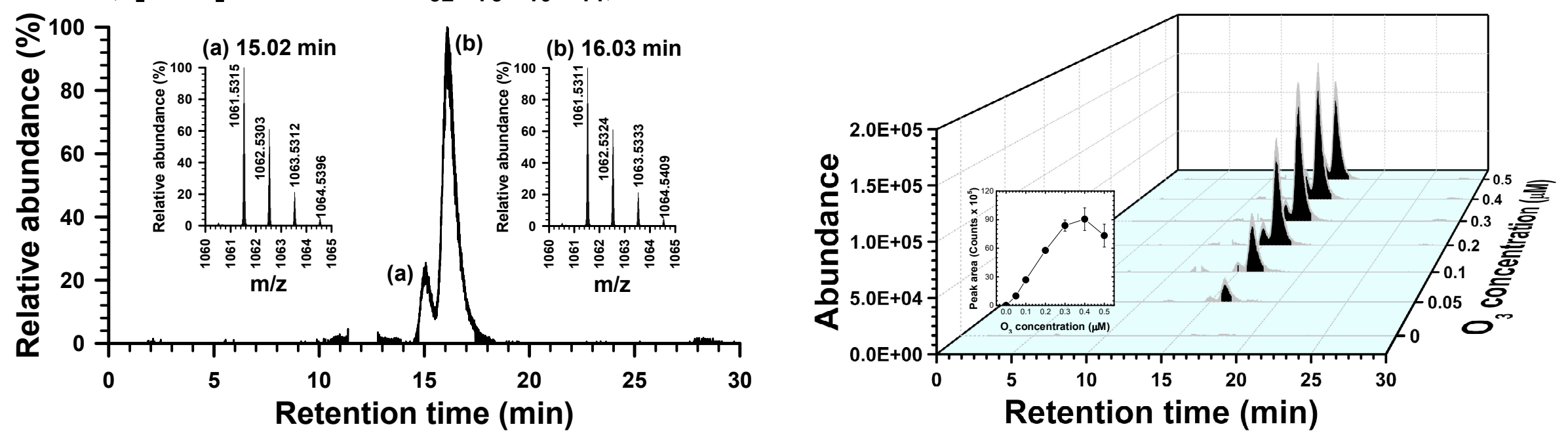

Figure S37. LC/MS identification of PYR6 $\left([\mathrm{M}+\mathrm{H}]^{+}=1061.5302\right)$. The chromatogram at $0.4 \mu \mathrm{M}$ of $\mathrm{O}_{3}$ with mass spectra $(\mathrm{left})$ and $\mathrm{O}_{3}$ concentration-dependent chromatograms (right) of PYR6. 
PYR7, [M+H] ${ }^{+}$formula: $\mathrm{C}_{52} \mathrm{H}_{73} \mathrm{~N}_{10} \mathrm{O}_{15}$, Theoretical m/z: 1077.5251
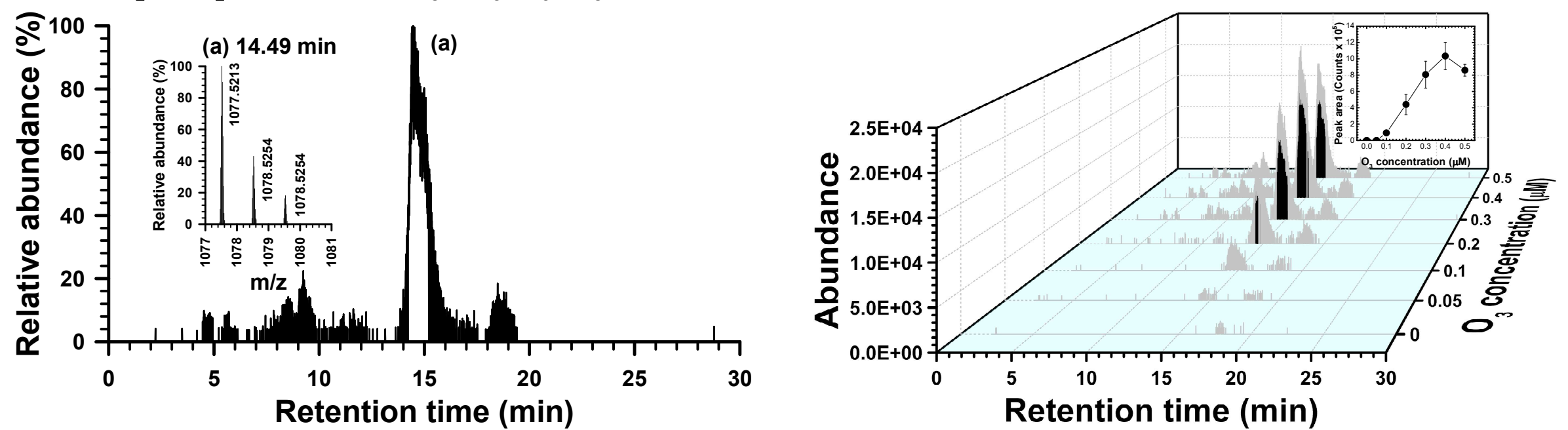

Figure S38. LC/MS identification of PYR7 $\left([\mathrm{M}+\mathrm{H}]^{+}=1077.5251\right)$. The chromatogram at $0.4 \mu \mathrm{M}$ of $\mathrm{O}_{3}$ with mass spectra $(\mathrm{left})$ and $\mathrm{O}_{3}$ concentration-dependent chromatograms (right) of PYR7. 
MC-LW, [M+H] $]^{+}$formula: $\mathrm{C}_{54} \mathrm{H}_{73} \mathrm{~N}_{8} \mathrm{O}_{12}$, Theoretical m/z: 1025.5342
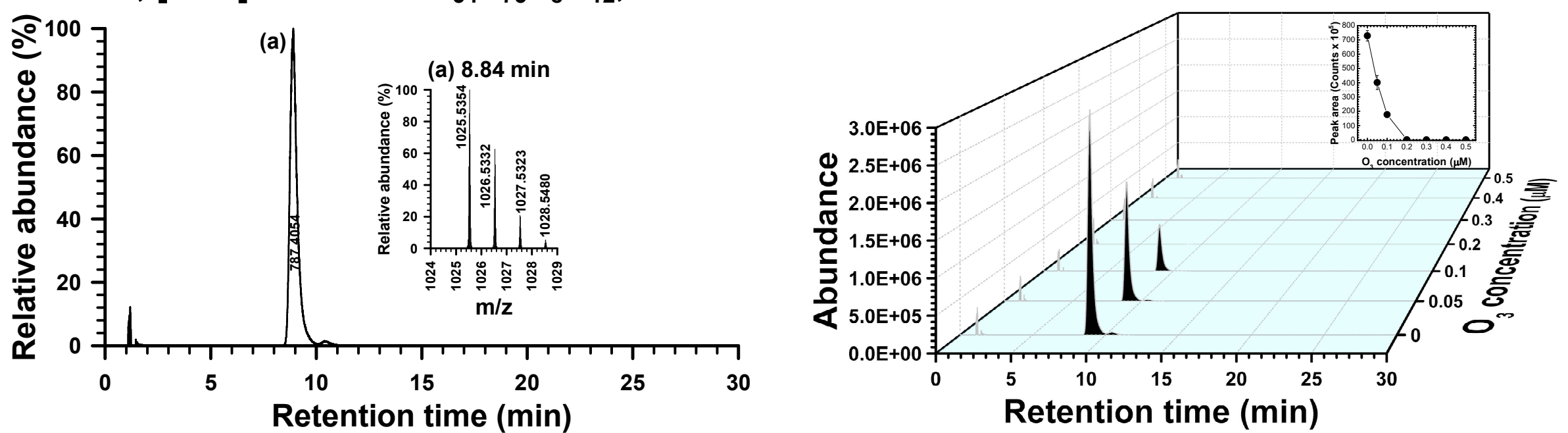

Figure S39. $\mathrm{LC} / \mathrm{MS}$ identification of MC-LW $\left([\mathrm{M}+\mathrm{H}]^{+}=1025.5342\right)$. The chromatogram at $0 \mu \mathrm{M}$ of $\mathrm{O}_{3}$ with mass spectra (left) and $\mathrm{O}_{3}$ concentration-dependent chromatograms (right) of MC-LW. 
PLW1, [M+H] $]^{+}$formula: $\mathrm{C}_{38} \mathrm{H}_{53} \mathrm{~N}_{8} \mathrm{O}_{14}$, Theoretical m/z: 845.3675
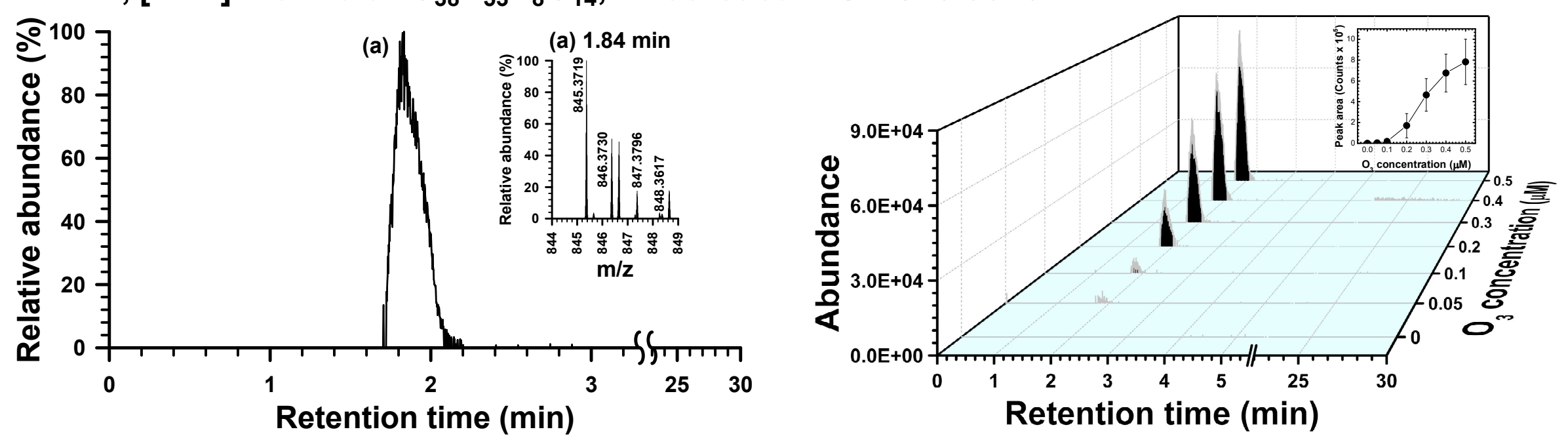

Figure S40. LC/MS identification of PLW1 $\left([\mathrm{M}+\mathrm{H}]^{+}=845.3675\right)$. The chromatogram at $0.5 \mu \mathrm{M}$ of $\mathrm{O}_{3}$ with mass spectra (left) and $\mathrm{O}_{3}$ concentration-dependent chromatograms (right) of PLW1. 
PLW2, $[\mathrm{M}+\mathrm{H}]^{+}$formula: $\mathrm{C}_{39} \mathrm{H}_{53} \mathrm{~N}_{8} \mathrm{O}_{15}$, Theoretical m/z: 873.3624
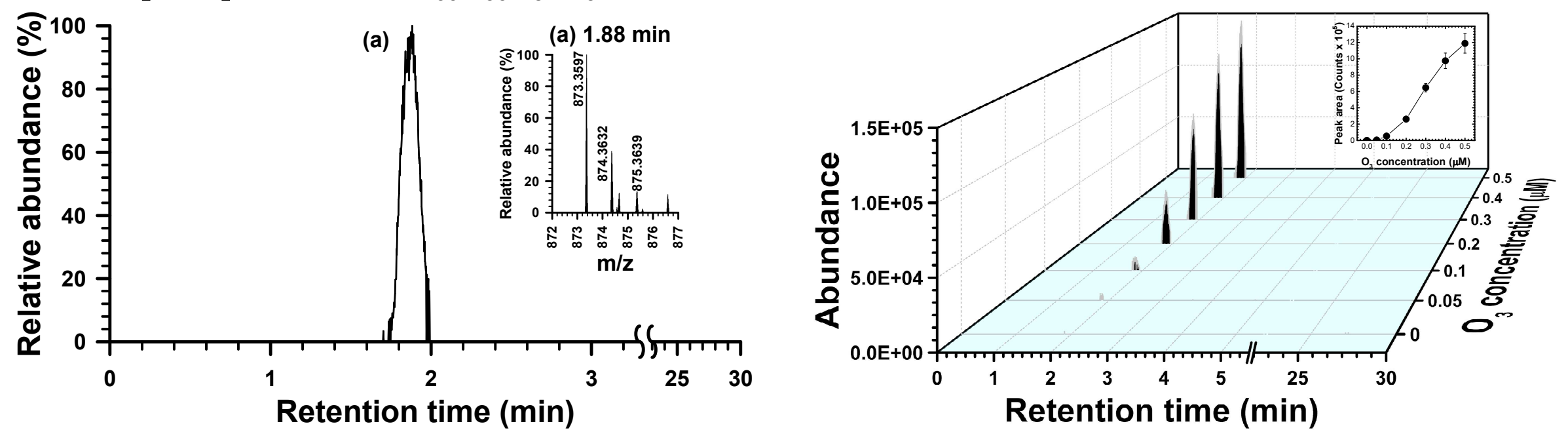

Figure S41. LC/MS identification of PLW2 $\left([\mathrm{M}+\mathrm{H}]^{+}=873.3624\right)$. The chromatogram at $0.5 \mu \mathrm{M}$ of $\mathrm{O}_{3}$ with mass spectra (left) and $\mathrm{O}_{3}$ concentration-dependent chromatograms (right) of PLW2. 
PLW3, [M+H] $]^{+}$formula: $\mathrm{C}_{41} \mathrm{H}_{57} \mathrm{~N}_{8} \mathrm{O}_{14}$, Theoretical m/z: 885.3988
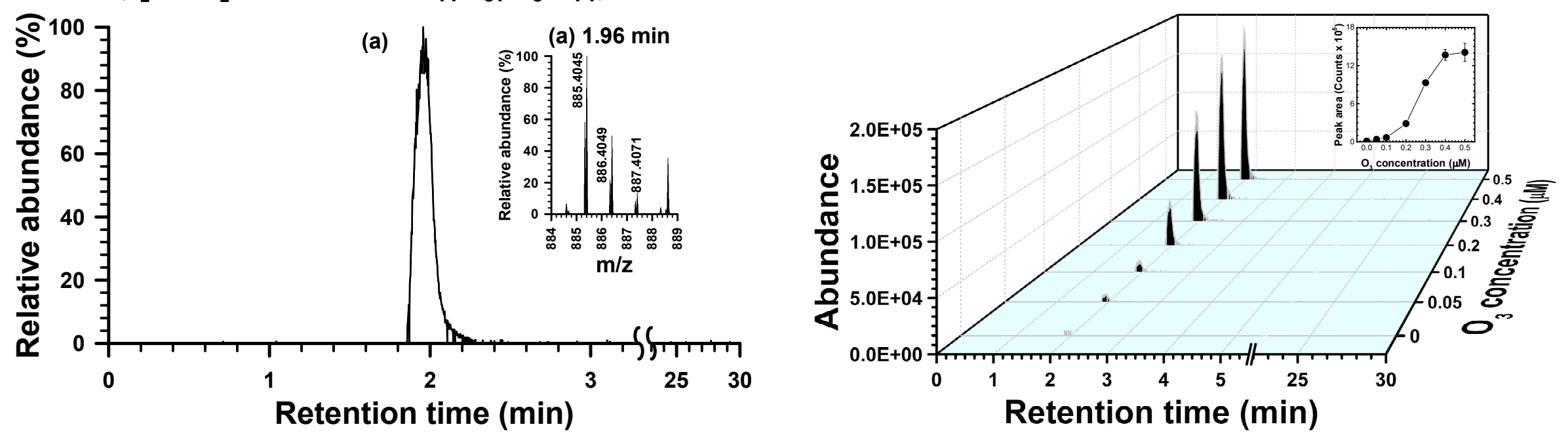

Figure S42. LC/MS identification of PLW3 $\left([\mathrm{M}+\mathrm{H}]^{+}=885.3988\right)$. The chromatogram at $0.5 \mu \mathrm{M}$ of $\mathrm{O}_{3}$ with mass spectra (left) and $\mathrm{O}_{3}$ concentration-dependent chromatograms (right) of PLW3. 
PLW4, [M+H] $]^{+}$formula: $\mathrm{C}_{53} \mathrm{H}_{73} \mathrm{~N}_{8} \mathrm{O}_{13}$, Theoretical m/z: 1029.5291
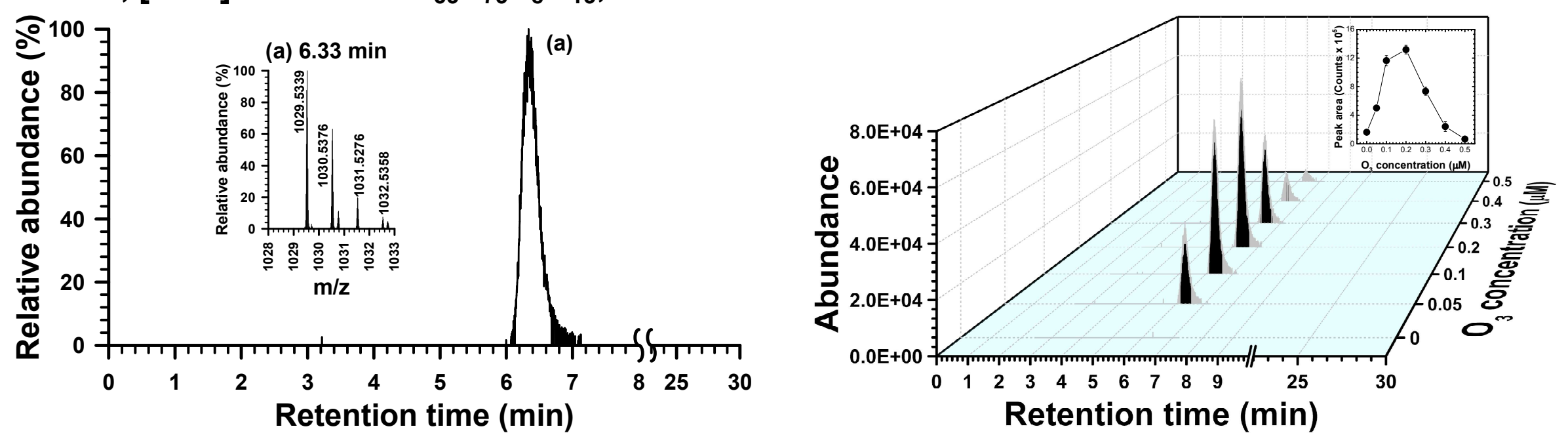

Figure S43. LC/MS identification of PLW4 $\left([\mathrm{M}+\mathrm{H}]^{+}=1029.5291\right)$. The chromatogram at $0.2 \mu \mathrm{M}$ of $\mathrm{O}_{3}$ with mass spectra $(\mathrm{left})$ and $\mathrm{O}_{3}$ concentration-dependent chromatograms (right) of PLW4. 
PLW5, [M+H] ${ }^{+}$formula: $\mathrm{C}_{53} \mathrm{H}_{73} \mathrm{~N}_{8} \mathrm{O}_{14}$, Theoretical m/z: 1045.5240
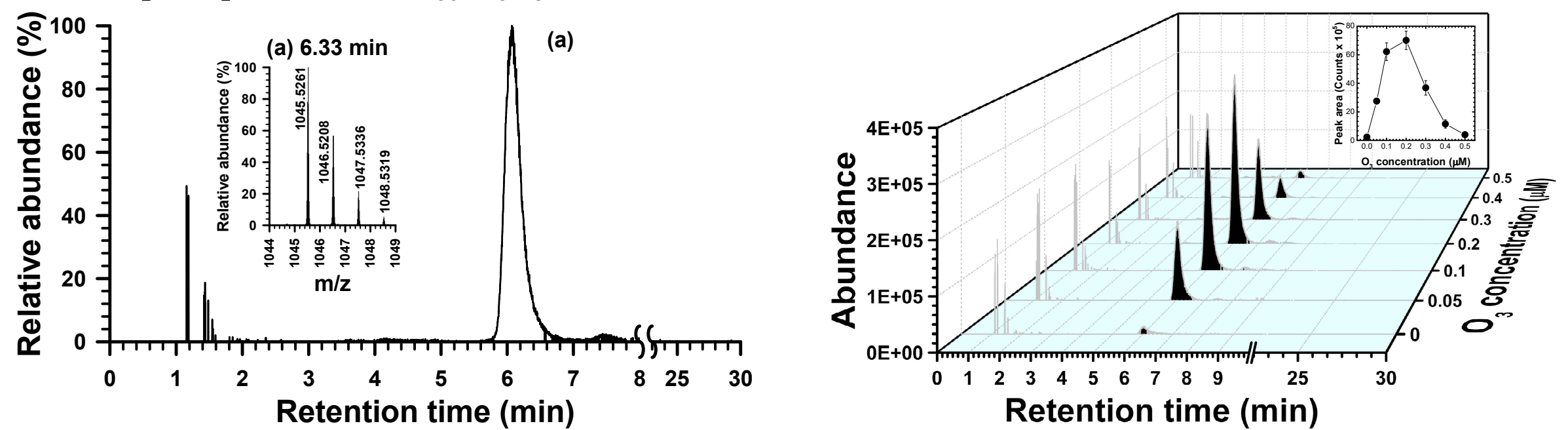

Figure S44. LC/MS identification of PLW5 $\left([\mathrm{M}+\mathrm{H}]^{+}=1045.5240\right)$. The chromatogram at $0.2 \mu \mathrm{M}$ of $\mathrm{O}_{3}$ with mass spectra (left) and $\mathrm{O}_{3}$ concentration-dependent chromatograms (right) of PLW5. 
PLW6, [M+H] $]^{+}$formula: $\mathrm{C}_{54} \mathrm{H}_{73} \mathrm{~N}_{8} \mathrm{O}_{14}$, Theoretical m/z: 1057.5240
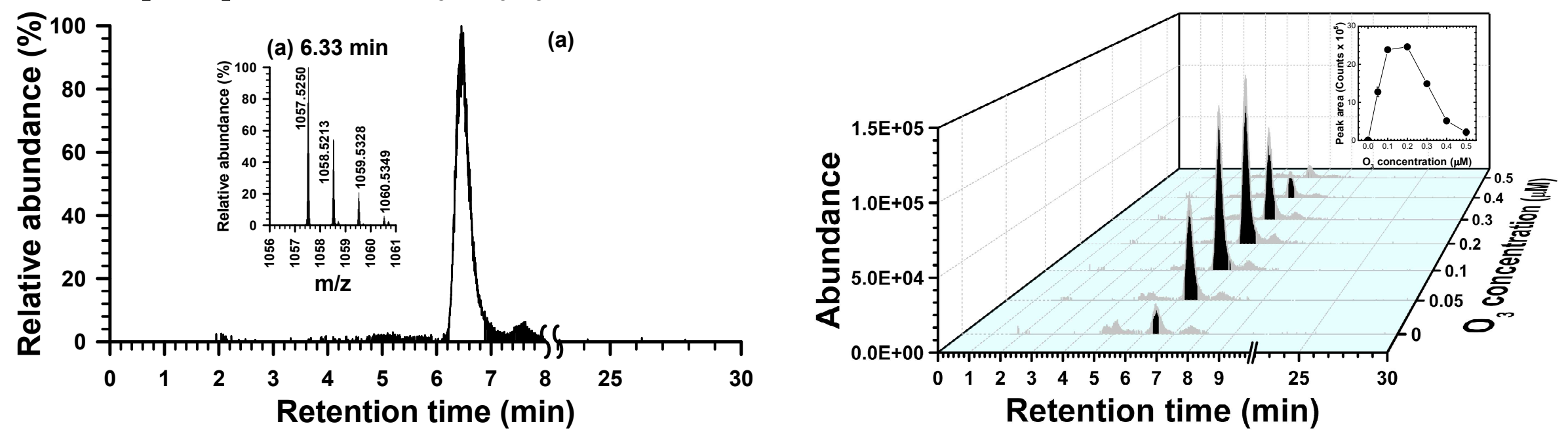

Figure S45. LC/MS identification of PLW6 $\left([\mathrm{M}+\mathrm{H}]^{+}=1057.5240\right)$. The chromatogram at $0.2 \mu \mathrm{M}$ of $\mathrm{O}_{3}$ with mass spectra (left) and $\mathrm{O}_{3}$ concentration-dependent chromatograms (right) of PLW6. 
PLW7, [M+H] $]^{+}$formula: $\mathrm{C}_{54} \mathrm{H}_{73} \mathrm{~N}_{8} \mathrm{O}_{15}$, Theoretical m/z: 1073.5189
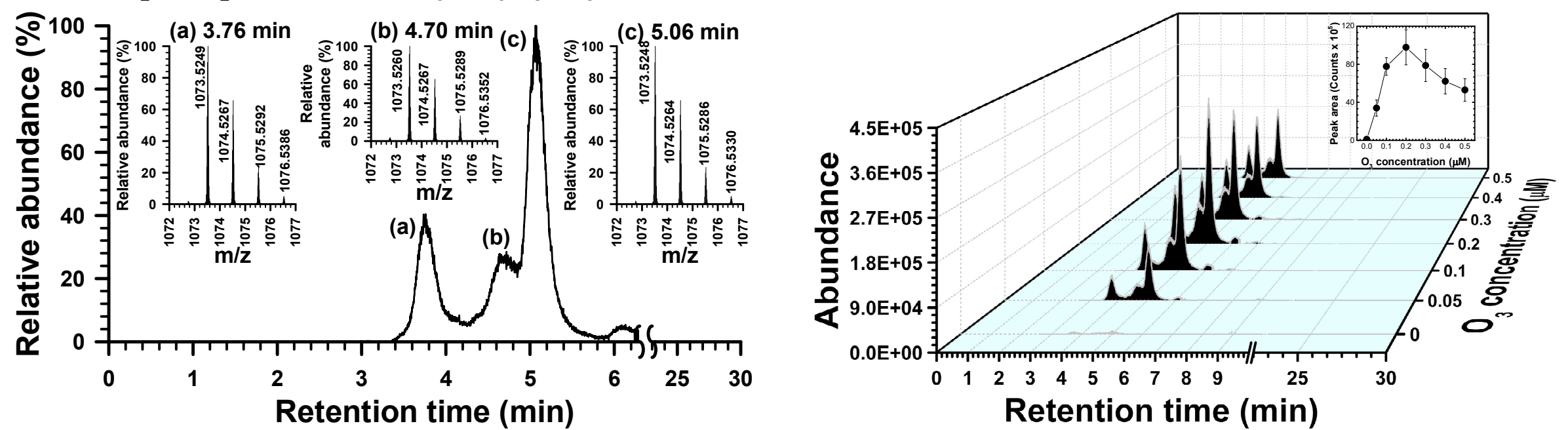

Figure S46. LC/MS identification of PLW7 $\left([\mathrm{M}+\mathrm{H}]^{+}=1073.5189\right)$. The chromatogram at $0.2 \mu \mathrm{M}$ of $\mathrm{O}_{3}$ with mass spectra $($ left $)$ and $\mathrm{O}_{3}$ concentration-dependent chromatograms (right) of PLW7. 
(a) Alkene; Reaction I (RI; Criegee mechanism)

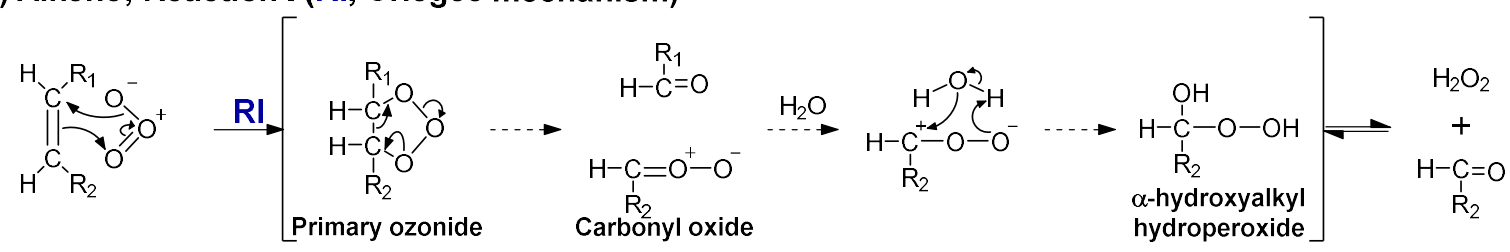

(b) Phenolic group; Reactions I, II and III (RI, RII and RIII)
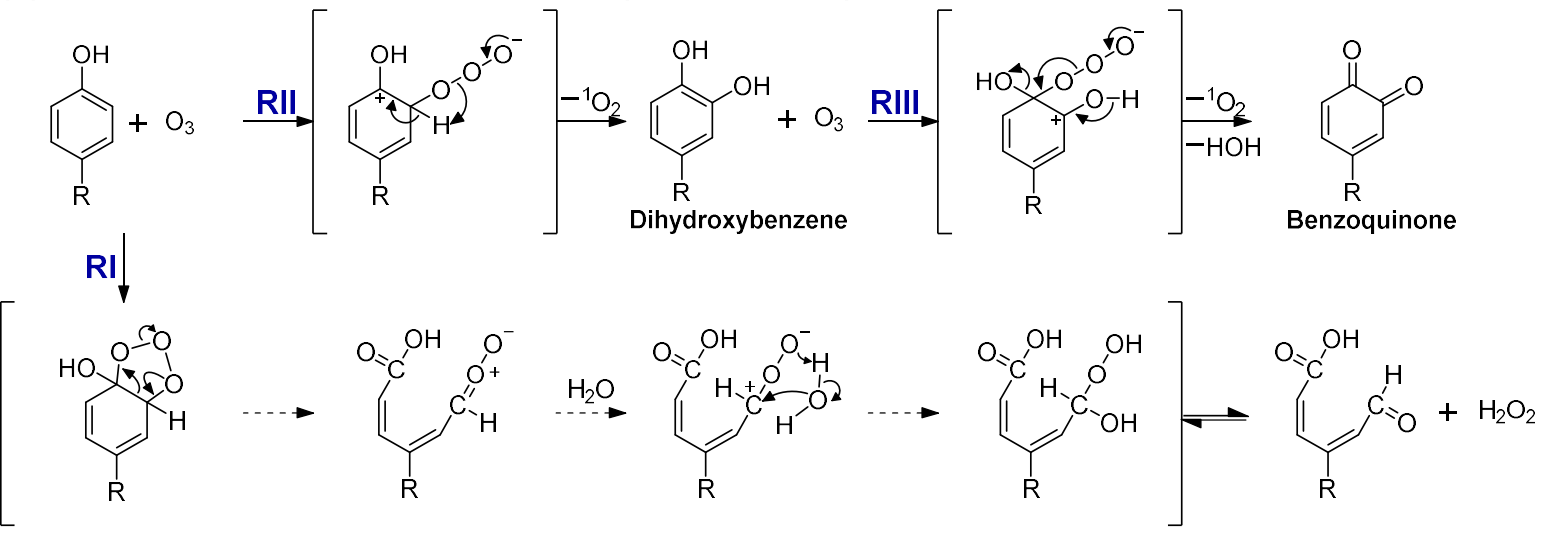

(c) Tryptophan (indolic double bond); Reactions I, II and IV (I, II and IV)

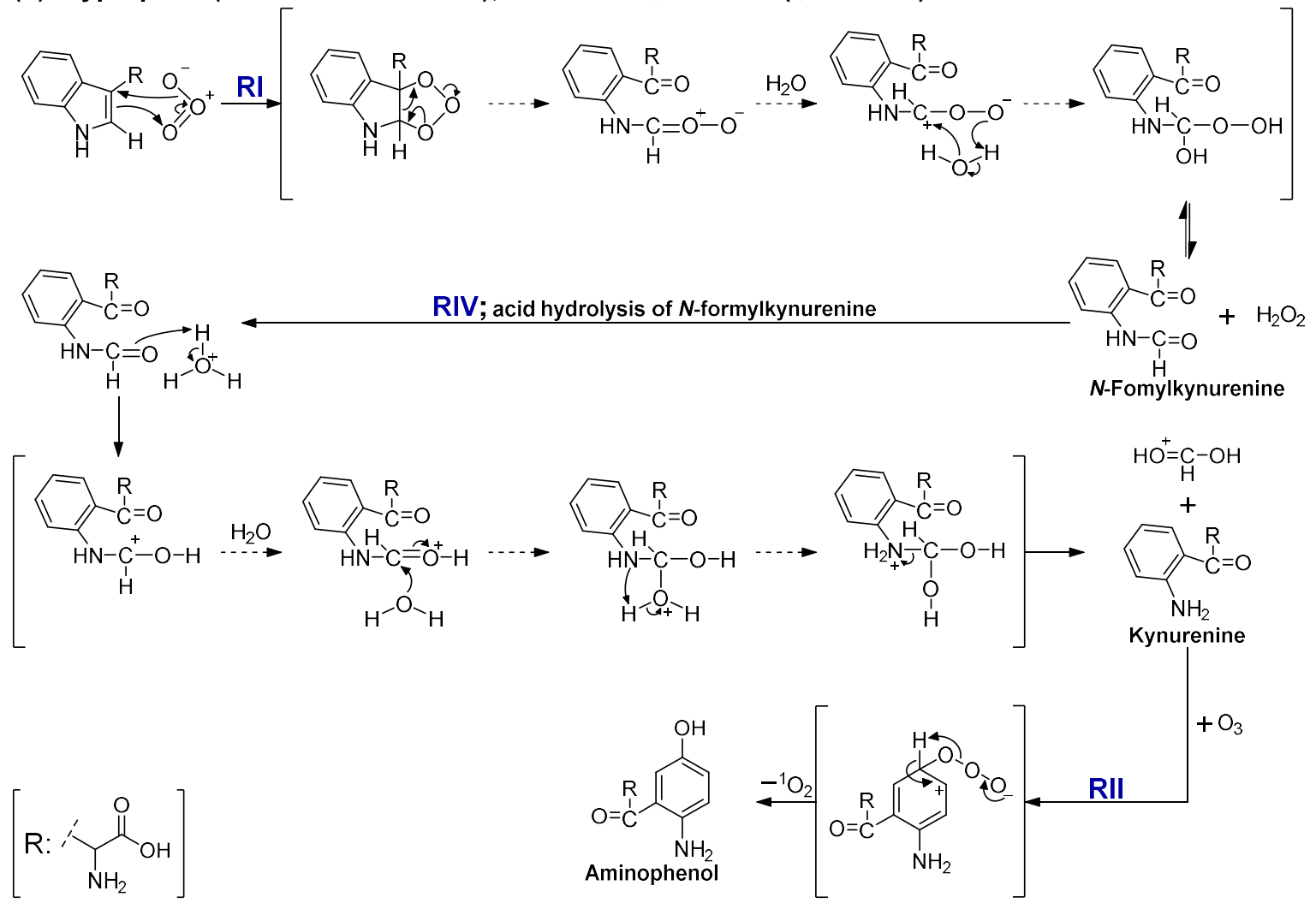

Figure S47. Reaction mechanisms of ozonation of major functional groups in MCs. 


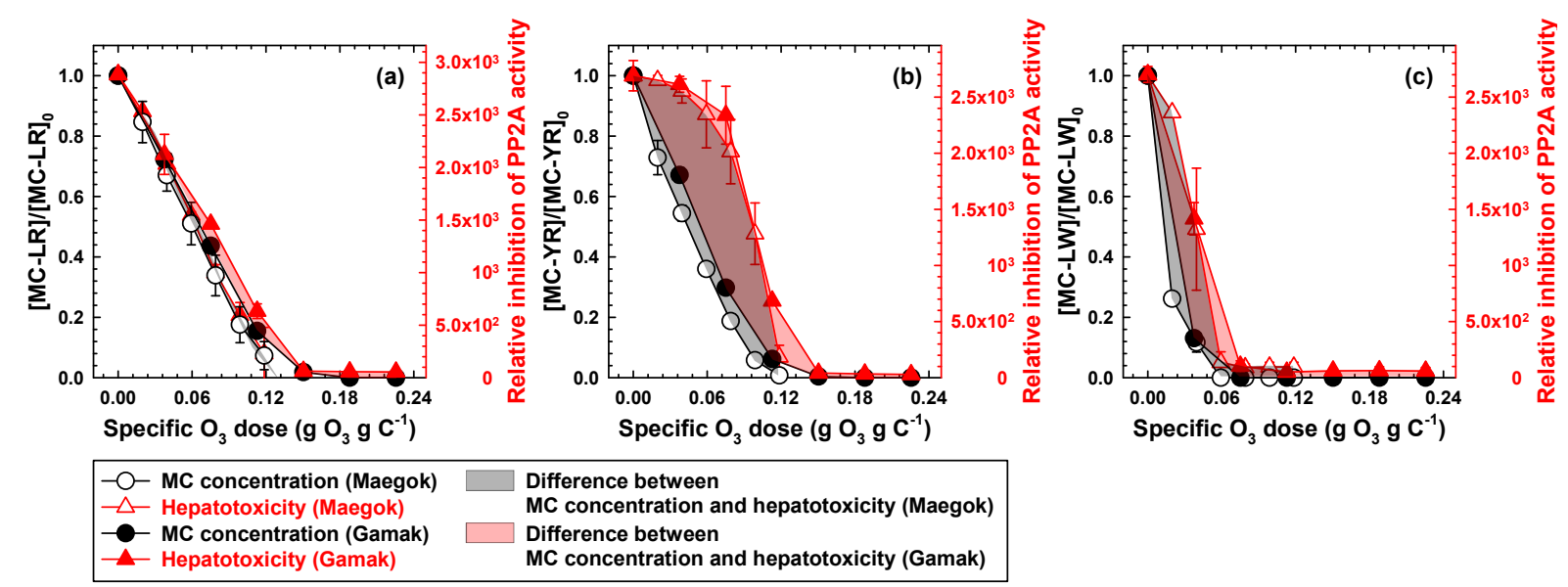

Figure S48. Changes of MC concentration and hepatotoxicity by reaction with $\mathrm{O}_{3}$ at different doses in natural waters $\left([\mathrm{MCs}]_{0}=0.1 \mu \mathrm{M}\right.$, temperature $\left.=20 \pm 1^{\circ} \mathrm{C}\right)$. 


\section{References}

S1. Moffitt, M. C.; Louie, G. V.; Bowman, M. E.; Pence, J.; Noel, J. P.; Moore, B. S. Discovery of two cyanobacterial phenylalanine ammonia lyases: Kinetic and structure characterization. Biochem. 2007, 46 (4), 1004-1012.

S2. Elovitz, M. S.; von Gunten, U. Hydroxyl radical/ozone ratios during ozonation processes. I. The $\mathrm{R}_{c t}$ concept. Ozone Sci. Eng. 1999, 21 (3), 239-260.

S3. Jia, Z.; Ramstad, T.; Zhong, M. Medium-throughput $\mathrm{p} K_{\mathrm{a}}$ screening of pharmaceuticals by pressure-assisted capillary electrophoresis. Electrophoresis 2001, 22 (6), 1112-1118.

S4. Neta, P.; Dorfman, L. M. Pulse radiolysis sduties. XIII. Rate constants for the reaction of hydroxyl radicals with aromatic compounds in aqueous solutions. In Advances in Chemistry, Radiation Chemistry; American Chemical Society, 1968; Vol. 81, pp 222230.

S5. Ashton, L.; Buxton, G. V.; Stuart, C. R. Temperature dependence of the rate of reaction of $\mathrm{OH}$ with some aromatic compounds in aqueous solution. J. Chem. Soc. Faraday Trans. 1995, 91 (11), 1631-1633.

S6. Baxendale, J. H.; Wilson, J. A. The photolysis of hydrogen peroxide at high light intensities. Trans. Faraday Soc. 1957, 53, 344-356. 